PNL -4293

I

NUPEG/CR-2881

PNL-4293

\title{
Experiment Operations Plan for the MT-4 Experiment in the NRU Reactor
}

Prepared by G. E. Russcher, C. L. Wilson, L. J. Parchen, R. K. Marshall, G. M. Hesson, B. J. Webb, M. D. Freshley

Pacific Northwest Laboratory

Operated by

Battelle Memorial Institute

\section{Prepared for}

U.S. Nuclear Regulatory

Commission 


\section{NOTICE}

This report was prepared as an account of work sponsored by an agency of the United States Government. Neither the United States Government nor any agency thereof, or any of their employees, makes any warranty, expressed or implied, or assumes any legal liability of responsibility for any third party's use, or the results of such use, of any information, apparatus, product or process disclosed in this report, or represents that its use by such third party would not infringe privately owned rights.

\section{Availability of Reference Materials Cited in NRC Publications}

Most documents cited in NRC publications will be available from one of the following sources:

1. The NRC Public Document Room, 1717 H Street, N.W. Washington, DC 20555

2. The NRC/GPO Sales Program, U.S. Nuclear Regulatory Commission, Washington, DC 20555

3. The National Technical Information Service, Springfield, VA 22161

Although the listing that follows represents the majority of documents cited in NRC publications, it is not intended to be exhaustive.

Referenced documents available for inspection and copying for a fee from the NRC Public Document Room include NRC correspondence and ir,ternal NRC memoranda; NRC Office of Inspection and Enforcement bulletins, circulars, information notices, inspection and investigation notices; Licensee Event Reports; vendor reports and correspondence; Commission papers; and applicant and licensee documents and correspondence.

The following documents in the NUREG series are available for purchase from the NRC/GPO Sales Program: formal NRC staff and contractor reports, NRC-sponsored conference proceedings, and NRC booklets and brochures. Also available are Regulatory Guides, NRC regulations in the Code of Federal Regulations, and Nuclear Regulatory Commission /ssuances.

Documents available from the National Technical Information Service include NUREG series reports and technical reports prepared by other federal agencies and reports prepared by the Atomic Energy Commission, forerunner agency to the Nuclear Regulatory Commission.

Documents available from public and special technical libraries include all open literature items, such as books, journal and periodical articles, and transactions. Federal Register notices, federal and state legislation, and congressional reports can usually be obtained from these libraries.

Documents such as theses, dissertations, foreign reports and transiations, and non-NRC conference proceedings are available for purchase from the organization sponsoring the publication cited.

Single copies of NRC draft reports are available free upon written request to the Division of Technical Information and Document Control, U.S. Nuclear Regulatory Commission, Washington, DC 20555.

Copies of industry codes and standards used in a substantive manner in the NRC regulatory process are maintained at the NRC Library, 7920 Norfolk Avenue, Bethesda, Maryland, and are available there for reference use by the public. Codes and standards are usually copyrighted and may be purchased from the originating organization or, if they are American National Standards, from the American National Standards Institute, 1430 Broadway, New York, NY 10018. 
NUREG/CR-2881

PNL-4293

R3

\section{Experiment Operations Plan for the MT-4 Experiment in the NRU Reactor}

Manuscript Completed: May 1983

Date Published; June 1983

Prepared by

G. E. Russcher, C. L. Wilson, L. J. Parchen,

R. K. Marshall, G. M. Hesson, B. J. Webb,

M. D. Freshiey

Pacific Northwest Laboratory

Richland, WA 99352

\section{Prepared for}

Division of Accident Evaluation

Office of Nuclear Regulatory Reseerch

U.S. Nuclear Regulatory Commission

Washington, D.C. 20555

NRC FIN B2277 



\section{ABSTRACT}

A series of thermal-hydraulic and cladding materials deformation experiments were conducted using light-water reactor fuel bundles as part of the Pacific Northwest Laboratory Loss-of-Coolant Accident (LOCA) Simulation Program. This report is the formal operations plan for MT-4--the fourth materials deformation experiment conducted in the National Research Universal (NRU) reactor, Chalk River, Ontario, Canada. A major objective of MT-4 was to simulate a pressurized water reactor LOCA that could induce fuel rod cladding deformation and rupture due to a short-term adiabatic transient and a peak fuel cladding temperature of $1200 \mathrm{~K}\left(1700^{\circ} \mathrm{F}\right)$. 



\section{SUMMARY}

The Loss-of-Coolant Accident (LOCA) Simulation Program was conducted by Pacific Northwest Laboratory (PNL) to evaluate the thermal-hydraulic and mechanical deformation behavior of full-length light-water reactor (LWR) fuel bundles under LOCA conditions. The test conditions were designed to simulate the heatup, reflood, and quench phases of a large-break LOCA; and the experiments were performed in the National Research Universal (NRU) reactor using nuclear fission to simulate the low-level decay power that is typical of these conditions.

The formal experiment operations plan for the seventh experiment in this program--materials experiment 4 (MT-4)--is presented in this document. The MT-4 experiment simulated a LOCA that produced a peak cladding temperature of $1200 \mathrm{~K}\left(1700^{\circ} \mathrm{F}\right)$ during a relatively rapid (adiabatic) heatup transient. Experiment operations closely followed the operating conditions used in TH-1.16, where reflood cooling was used to turn around the heatup transient caused by a long reflood delay. Afterward, thermal-hydraulic measurements evaluated the effects of fuel cladding deformation and rupture.

An approved draft copy of the experiment operations plan for MT-4 was sent to Chalk River Nuclear Laboratories (CRNL), Chalk River, Ontario, in May 1982 just prior to the MT-4 experiment. This report represents the formal documentation of the experiment operations plan for MT-4. 



\section{CONTENTS}

ABSTRACT...................................................... i i

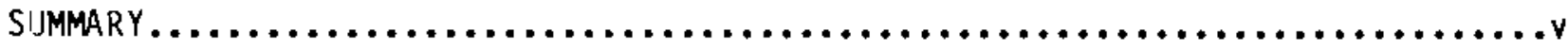

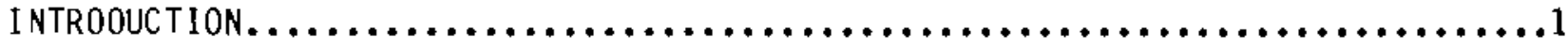

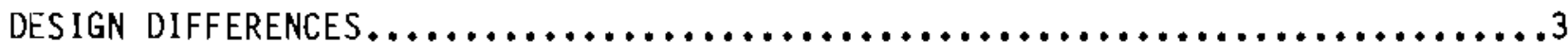

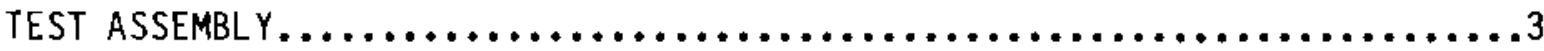

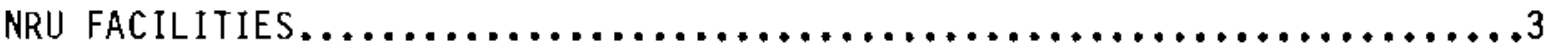

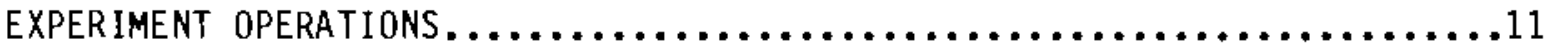

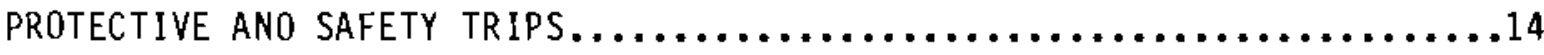

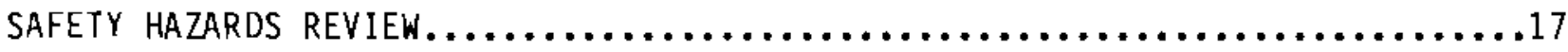

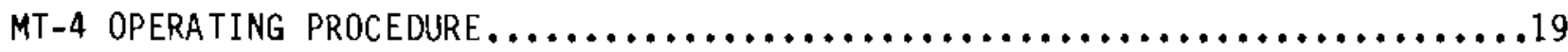

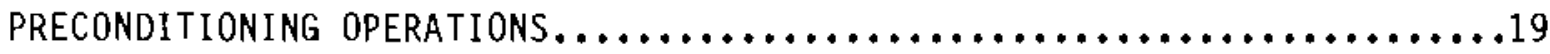

REFLOOD CALIBRATION, PRETRANSIENT, AND TRANSIENT OPERATIONS.........23

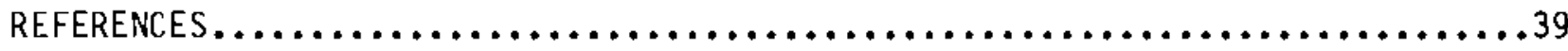

APPENUIX A - TEST PARAMETER LOG...................................

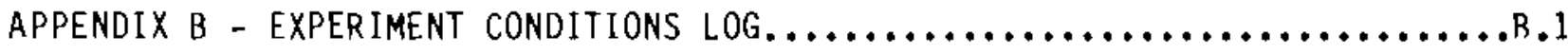





\section{TABLES}

1 DACS and Instrumentation Array for MT-4..........................4

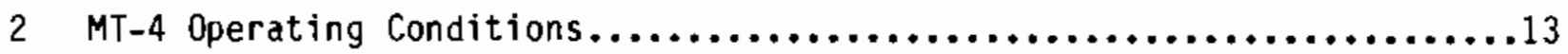

3 Pretransient and Transient Safety Trip Set Points...................15

4 Standard and Defaulted Safety Trip Set Point Criteria for Operating and Nonfunctional Fuel Cladding Thermocouples...............16

5 Water Chemistry Requirements..................................

6 U-2 Loop Instrument Calibration...............................20

7 Preconditioning Safety Trip Set Points........................... 20

8 MT-4 Operating Conditions........................................24

9 Steam/Reflood Loop Calibration...............................25

10 Pretransient and Transient Safety Trip Set Points...................27

11 Standard and Defaulted Safety Trip Set Point Criteria for Operating and Nonfunctional Fuel Cladding Thermocouples..............28

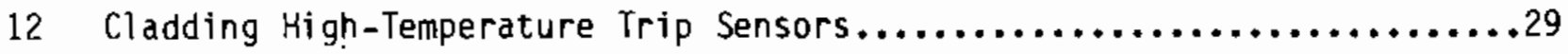

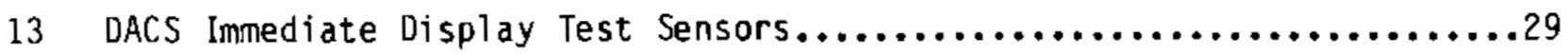

14 Adiabatic Experiment Conditions Log...........................33

15 LOCA Experiment Conditions Log................................. 35 


\section{$\cdot$}




\section{INTRODUCTION}

The Loss-of-Coolant Accident (LOCA) Simulation Program was conducted in the National Research Universal (NRU) reactor at Chalk River Nuclear Laboratories (CRNL), (a) Chalk River, Ontario, Canada, by Pacific Northwest Laboratory (PNL). (D) The program was sponsored by the U.S. Nuclear Regulatory Commission (NRC) to evaluate the thermal-hydraulic and mechanical deformation behavior of full-length, $3 \%$ enriched light-water reactor (LWR) fuel rod bundles during the heatup, reflood, and quench phases of a LOCA. Low-level nuclear fission heat was used to simulate the decay heat in the fuel and cladding that is typical of a LOCA. (1)

Previous materials deformation experiments in the L.OCA program emphasized higher temperature LOCA fuel failure and extended operating time at cladding deformation conditions. The MT-2 experiment produced ruptured fuel rods during a simulated LOCA transient in which peak fuel rod cladding temperatures exceeded $1200 \mathrm{~K}\left(1700^{\circ} \mathrm{F}\right)$. MT-3 produced maximum ballooned and ruptured fuel rods during an extended LOCA transient in the Zircaloy high alpha temperature regime.

The MT-4 experiment is most similar to the MT-2 experiment, except for three differences: 1) the MT-4 heatup was a short-term adiabatic transient rather than an extended one, 2) after the adiabatic heatup transient and temperature turnaround, peak cladding temperatures were stabilized to measure heat transfer characteristics of the deformed (and ruptured) fuel rods, and 3) selfpowered neutron detectors (SPNDs) mounted on the shroud were relocated to grid elevations to minimize axial neutron power distortion.

The major objectives of the MT-4 experiment were to:

- evaluate simulated LOCA fuel rupture during adiabatic heatup conditions

- evaluate LOCA fuel rupture in a carefully monitored neutronic environment

- compare MT-4 results with those from the MT-2 and MT-3 experiments (all other variables were minimized)

- simulate LOCA fuel rupture (12 rods) during a delayed reflooding transient with a peak cladding temperature of $1200 \mathrm{~K}\left(1700^{\circ} \mathrm{F}\right)$

(a) Operated by Atomic Energy of Canada Ltd. (AECL).

(b) Operated for the U.S. Department of Energy (DOE) by Battelle Memorial Institute. 
- measure heat transfer coefficients for deformed fue 1 rods at $1089 \mathrm{~K}$ and $1200 \mathrm{~K}\left(1500^{\circ} \mathrm{F}\right.$ and $\left.1700^{\circ} \mathrm{F}\right)$.

The MT-4 experiment used a new cruciform bundle of 12 pressurized test fuel rods and the 20 guard fuel rods and the shroud that were previously used in MT-3. Test operations most closely followed the operating conditions of TH-1.16, where reflood cooling was used to terminate the heatup transient temperature at $\sim 1200 \mathrm{~K}\left(1700^{\circ} \mathrm{F}\right)$. Stabilized post-transient operation closely followed the operating conditions used in the MT-3 experiment to investigate hightemperature thermal-hydraulic effects of the LOCA.

This report presents the formal experiment operations plan for the fourth materials deformation experiment in the program (MT-4), which was performed in May 1982. The remainder of the report consists of:

- a summary of the design differences for MT-4 as. compared with MT-3

- a safety hazards statement

- the operating procedure for MT-4

- a sample Test Parameter Log (Appendix A) and an Experiment Conditions Log (Appendix B). 


\section{DESIGN DIFFERENCES}

The MT-4 test assembly was quite similar to the MT-3 test assembly; therefore, only design differences are described in this report. Detailed descriptions are given in the safety analysis report(2) and the experiment operations plan for MT-3. (3)

\section{TEST ASSEMBLY}

The MT-4 test assembly used a new cruciform bundle of 12 test fuel rods and the MT-3 guard fuel rod bundle and shroud. The 12 fuel rods were pressurized to $4.62 \mathrm{MPa}$ (670 psia) just prior to the transient and were monitored by pressure transducers located just above the reactor head. Guard fuel rods were pressurized to $0.101 \mathrm{MPa}(14.7 \mathrm{psia})$.

Because the MT-3 guard fuel rod assembly had been used in only one previous experiment, most instrumentation was still operational for this test. (2) In addition, three new types of instrumentation were included in MT-4: 1) a new 1 iquid level sensor that sampled the coolant at Level (18) 139.9 ; (a) 2) a time domain reflectometer (TDR) sensor that monitored the coolant liquid level in the test assembly; and 3) cumulative neutron flux (fluence) monitor wires. Most of the instrumentation that had been added for the MT-3 experiment remained: 1) two thermocouples (TCs) at fuel rod braze junctions at Level (19) 155.7 ; 2) two more TCs on the hanger tube at Level (20) 168.7 ; 3) one TC in a fuel rod plenum region; and 4) the desuperheater spray coolant system.

The MT-4 test rod bundle with new fuel rods and instrumentation constituted the cruciform test fuel assembly. The 12 test rods were pressurized to 4.62 MPa (670 psia) with helium just before the LOCA experiment and were instrumented the same as those in the MT-3 assembly. Fuel rod 4D, which replaced the instrument tube, also had one TC mounted in the fission gas plenum. The test rod instrumentation is summarized in Table 1; new data acquisition and control system (DACS) terminal numbers, names, and sensor locations in the test train are given.

\section{NRU FACILITIES}

The NRU reactor and loops were basically unchanged from previous LOCA experiments. $(3,4)$ The same reactor fuel loading was used to provide the

(a) Instrument levels are reported with the old designation in parentheses followed by the new number (which indicates the inches above the top of the tie plate of the test assembly). 
TABLE 1. DACS and Instrumentation Array for MT-4

\begin{tabular}{c}
$\begin{array}{c}\text { Instrument } \\
\text { Level }\end{array}$ \\
\hline
\end{tabular}

\begin{tabular}{|c|c|}
\hline (01d) & New \\
\hline (1) & \\
\hline
\end{tabular}

(1) $\quad-0.2 \quad 106 \quad$ TC-(-)0.2-1F-IN

(2) $8.7^{\text {(a) }} \quad 74 \quad$ TC-08.7-6F-C -4

(2) $8.7 \quad 4 \quad$ TC-08.7-6F-S-C

(2) $8.7 \quad 82 \quad$ TC-08.7-6A-C-3

(2) $8.7 \quad 5 \quad$ TC-08.7-1A-S-C

(3) 13.9 (a) $83 \quad T C-13.9-6 F-C-4$

(3) $13.9 \quad 11 \quad T C-13.9-6 \mathrm{~A}-\mathrm{C}-3$

(3) $13.9 \quad 86 \quad$ TC-13.9-6A-S-C

(3) $13.9 \quad 12 \quad$ TC-13.9-1F-S-C

(4) $19.2 \quad 13 \quad$ TC-19.2-6F-S-C

(4) $19.2 \quad 14 \quad$ TC-19.2-1A-S-C

(5) $28.0 \quad 15 \quad$ TC $-28.0-6 \mathrm{~A}-\mathrm{S}-\mathrm{C}$

(5) $28.0 \quad 16 \quad$ TC $-28.0-1 \mathrm{~F}-\mathrm{S}-\mathrm{C}$

(6) $34.9 \quad 19 \quad$ TC-34.9-6F-S-C

(6) $34.9 \quad 18 \quad$ TC $-34.9-6 \mathrm{~A}-\mathrm{S}-\mathrm{C}$

(6) $34.9 \quad 21 \quad$ TC-34.9-1A-S-C

(6) $34.9 \quad 20 \quad$ TC-34.9-1F-S-C

(7) $40.2 \quad 35 \quad$ TC $-40.2-5 F-I R-4$

(7) $40.2 \quad 33$ TC-40.2-2A-IR-2 Failed in MT-4 preconditioning(b)

(8) $42.9 \quad 30 \quad T C-42.9-6 F-S-C$

(8) $42.9 \quad 31 \quad$ TC-42.9-1A-S-C

(9) $49.0 \quad 36 \quad$ TC $-49.0-6 \mathrm{~A}-\mathrm{S}-\mathrm{C}$

(9) $49.0 \quad 37 \quad$ TC-49.0-1F-S-C

(10) $55.9 \quad 40 \quad$ TC $-55.9-6 \mathrm{~F}-\mathrm{S}-\mathrm{C}$

(10) $55.9 \quad 39 \quad$ TC-55.9-6A-S-C

(10) $55.9 \quad 42 \quad$ TC $-55.9-1 \mathrm{~A}-\mathrm{S}-\mathrm{C}$

(10) $55.9 \quad 41 \quad$ TC-55.9-1F-S-C

(11) $61.2 \quad 47 \quad$ TC-61.2-6F-S-C

(11) $61.2 \quad 104 \quad$ TC-61.2-6B-IR-3 
IABLE 1. (contd)

\begin{tabular}{|c|c|c|c|c|}
\hline \multicolumn{2}{|c|}{$\begin{array}{c}\text { Instrument } \\
\text { Leve } 1\end{array}$} & \multirow{2}{*}{$\begin{array}{l}\text { DACS } \\
\text { Channel } \\
\text { Number } \\
\end{array}$} & \multirow[b]{2}{*}{ Sensor Name } & \multirow[b]{2}{*}{ Notes } \\
\hline$\overline{(01 d)}$ & New & & & \\
\hline (11) & 61.2 & 34 & TC-61.2-3A-IR-2 & \\
\hline (11) & 61.2 & 48 & $T C-61.2-1 \mathrm{~A}-\mathrm{S}-\mathrm{C}$ & \\
\hline (11) & 61.2 & 102 & TC-61.2-1E-IR-1 & \\
\hline (11) & 61.2 & 132 & TC-61.2-4F-IR-4 & \\
\hline$(12)$ & 69.0 & 59 & TC-69.0-5E-SP-4 & \\
\hline (12) & 69.0 & 58 & $T C-69.0-5 B-S P-3$ & \\
\hline (12) & 69.0 & 57 & $\mathrm{TC}-69.0-2 \mathrm{~B}-\mathrm{SP}-2$ & \\
\hline (12) & 69.0 & 56 & $\mathrm{TC}-69.0-2 \mathrm{E}-\mathrm{SP}-1$ & \\
\hline-- & 73.9 & 186 & $T C-73.9-50-1 R-2$ & Relocated (c) \\
\hline -- & 73.9 & 69 & TC-73.9-4D-IR-2 & Relocated \\
\hline-- & 73.9 & 95 & $T C-73.9-4 C-1 R-2$ & Relocated \\
\hline-- & 73.9 & 90 & TC $-73,9-30-1 R-2$ & Relocated \\
\hline (13) & 76.9 & 61 & $T C-76.9-6 \mathrm{~F}-\mathrm{S}-\mathrm{C}$ & \\
\hline (13) & 76.9 & 60 & TC-76.9-6A-S-C & \\
\hline (13) & 76.9 & 63 & TC-76.9-1A-S-C & \\
\hline (13) & 76.9 & 62 & TC-76.9-1F-S-C & \\
\hline (13) & 76.9 & $101^{(d)}$ & $T C-76.9-6 C-I R-3$ & PS-76.9-GR-IR-1 safety circuit \\
\hline (13) & 76.9 & $52(d)$ & TC-76.9-4A-IR-3 & PS-76.9-GR-IR-1 safety circuit \\
\hline (13) & 76.9 & 99 & TC-76.9-1D-IR-1 & \\
\hline (13) & 76.9 & ${ }_{50}(d)$ & $T C-76.9-3 F-I R-1$ & PS-76.9-GR-IR-1 safety circuit \\
\hline (13) & 76.9 & 53 & TC-76.9-5E-IR-4 & \\
\hline (13) & 76.9 & $67(d)$ & TC-76.9-5B-IR-1 & PS-76.9-GR-1R-1 safety circuit \\
\hline$(13\rangle$ & 76.9 & 23 & $T C-76.9-2 B-I R-2$ & \\
\hline (13) & 76.9 & 127 & $T C-76.9-2 C-I R-3$ & \\
\hline (13) & 76.9 & 135 & TC-76.9-2D-IR-3 & \\
\hline (13) & 76.9 & 32 & $T C-76.9-2 E-I R-3$ & \\
\hline (13) & 76.9 & 93 & TC-76.9-3E-IR-3 & \\
\hline (13) & 76.9 & 89 & TC-76.9-4E-IR-8 & \\
\hline-- & 78.9 & 123 & TC-78.9-5D-IR-3 & \\
\hline -- & 78.9 & 17 & TC-78.9-4D-IR-3 & \\
\hline -- & 78.9 & 88 & TC-78.9-4C-IR-3 & \\
\hline _. & 78.9 & 96 & $T C-78.9-30-I R-3$ & \\
\hline .- & 80.9 & 91 & TC-80.9-2C-IR-2 & Failed in MT-4 preconditioning(a) \\
\hline -- & 80.9 & 188 & TC-80.9-2D-IR-2 & \\
\hline -- & 80.9 & 38 & TC-80.9-3E-IR-2 & \\
\hline -- & 80.9 & 64 & TC-80.9-4E-IR-2 & \\
\hline
\end{tabular}


TABLE 1. (contd)

\begin{tabular}{|c|c|c|c|c|}
\hline \multicolumn{2}{|c|}{$\begin{array}{c}\text { Instrument } \\
\text { Level }\end{array}$} & \multirow{2}{*}{$\begin{array}{l}\text { DACS } \\
\text { Channel } \\
\text { Number } \\
\end{array}$} & \multirow[b]{2}{*}{ Sensor Name } & \multirow[b]{2}{*}{ Notes } \\
\hline (01d) & New & & & \\
\hline -- & 83.9 & 133 & TC-83.9-5C-IR-2 & \\
\hline -- & 83.9 & 117 & $T C-83.9-4 B-1 R-2$ & \\
\hline-- & 83.9 & 54 & TC-83.9-4D-IR-8 & \\
\hline-- & 83.9 & 137 & $T C-83.9-3 C-I R-2$ & Failed in MT-4 preconditioning(a) \\
\hline (14) & 90.0 & 65 & $T C-90.0-5 E-S P-4$ & \\
\hline (14) & 90.0 & 51 & $T C-90.0-5 B-S P-3$ & \\
\hline (14) & 90.0 & 44 & $T C-90.0-2 B-S P-2$ & Failed in MT- 3.06 \\
\hline (14) & 90.0 & 43 & TC-90.0-2E-SP-1 & \\
\hline -. & 93.9 & 115 & $T C-93.9-3 B-I R-2$ & \\
\hline-- & 93.9 & 49 & $T C-93.9-4 E-I R-3$ & \\
\hline (15) & 97.9 & $76(e)$ & TC-97.9-6F-S-C & PS-97-SH-5-2 protective circuit \\
\hline (15) & 97.9 & $75(\mathrm{e})$ & $T C-97.9-6 A-S-C$ & PS-97-SH-5-2 protective circuit \\
\hline (15) & 97.9 & $78(e)$ & $T C,-97.9-1 A-S-C$ & PS-97-SH-S-2 protective circuit \\
\hline (15) & 97.9 & $77(\mathrm{e})$ & TC-97.9-1F-S-C & PS-97-SH-S-2 protective circuit \\
\hline (15) & 97.9 & $103(f)$ & TC-97.9-1C-IR-2 & PS-97.9-GR-IR-1 safety circuit \\
\hline (15) & 97.9 & $25(f)$ & TC-97.9-5E-IR-2 & PS-97.9-GR-IR-1 safety circuit \\
\hline (15) & 97.9 & 10 & $T C-97.9-5 E-I R-C$ & \\
\hline (15) & 97.9 & $45(f)$ & $T C-97.9-5 B-I R-3$ & PS-97.9-GR-IR-1 safety circuit \\
\hline (15) & 97.9 & 46 & $T C-97.9-5 B-I R-C$ & \\
\hline (15) & 97.9 & $24(f)$ & TC-97.9-2B-IR-4 & PS-97.9-GR-IR-1 safety circuit \\
\hline (15) & 97.9 & 9 & TC-97.9-2B-IR-C & \\
\hline (15) & 97.9 & 55 & TC-97.9-2C-IR-8 & \\
\hline (15) & 97.9 & 119 & TC-97.9-2D-IR-8 & \\
\hline (15) & 97.9 & $2^{(f)}$ & $T C-97.9-2 E-[R-1$ & SD-97.9-IR-1 safety circuit \\
\hline (15) & 97.9 & 26 & TC-97.9-2E-IR-C & \\
\hline (15) & 97.9 & 122 & TC-97.9-3E-IR-8 & \\
\hline (15) & 97.9 & 105 & $T C-97.9-6 D-I R-4$ & Failed in MT-3 preconditioning \\
\hline-- & 101.9 & 135 & TC-1D1.9-5C-IR-3 & \\
\hline -- & 101.9 & 114 & $T C-101.9-4 B-I R-3$ & \\
\hline-- & 101.9 & 120 & TC-101.9-3B-IR-8 & \\
\hline -- & 101.9 & 190 & TC-101.9-3C-IR-3 & \\
\hline-- & 104.9 & 116 & $T C-104.9-5 C-1 R-8$ & \\
\hline -- & 104.9 & 1 & $T C=104.9-4 B-I R-8$ & \\
\hline-- & 104.9 & 94 & $T C-104.9-3 C-I R-8$ & \\
\hline (16) & 111.0 & 109 & $\mathrm{TC}-111.0-6 \mathrm{~F}-\mathrm{C}-4$ & \\
\hline (16) & 111.0 & 100 & $T C-111.0-6 A-C-3$ & \\
\hline
\end{tabular}




\section{TABLE 1. (contd)}

\begin{tabular}{|c|c|c|c|c|}
\hline \multicolumn{2}{|c|}{$\begin{array}{c}\text { Instrument } \\
\text { Level }\end{array}$} & \multirow{2}{*}{$\begin{array}{l}\text { DACS } \\
\text { Channel } \\
\text { Number } \\
\end{array}$} & \multirow[b]{2}{*}{ Sensor Name } & \multirow[b]{2}{*}{ Notes } \\
\hline (01d) & New & & & \\
\hline (16) & 111.0 & 107 & $T C-111.0-1 A-C-2$ & Failed in MT-3.06 \\
\hline$(16)$ & 111.0 & 98 & $T C-111.0-1 F-C-1$ & \\
\hline$(16)$ & 111.0 & 85 & $\mathrm{TC}-111,0-5 \mathrm{E}-\mathrm{SP}-4$ & \\
\hline$(16)$ & 111.0 & 84 & $T C-111.0-5 B-S P-3$ & \\
\hline (16) & 111.0 & $\mathrm{BO}$ & $\mathrm{TC}-111.0-2 \mathrm{~B}-\mathrm{SP}-2$ & \\
\hline$(16)$ & 111.0 & 72 & $\mathrm{TC}-111,0-2 \mathrm{E}-\mathrm{SP}-1$ & \\
\hline (16) & 111.0 & 98 & $T C-111.0-1 F-C-1$ & \\
\hline (17) & 118.9 & $111(\mathrm{e})$ & TC-118.9-6F-S-C & PS-118.9-SH-1 (b) protective circuit \\
\hline (17) & 118.9 & $110(e)$ & TC-118.9-6A-S-C & PS-118.9-SH-1 (b) protective circuit \\
\hline (17) & 118.9 & $113(\mathrm{e})$ & TC-118.9-1A-S-C & PS-118.9-SH-1 (b) protective circuit \\
\hline (17) & 118.9 & $112(\mathrm{e})$ & TC-118.9-1F-S-C & PS-118.9-SH-1 (b) protective circuit \\
\hline (17) & 118.9 & 68 & $T C-118.9-6 \mathrm{E}-\mathrm{IR}-4$ & Failed in MT-3.04 \\
\hline (17) & 118.9 & $81(9)$ & TC-118.9-1B-IR-2 & PS-118.9-GR-IR-1 safety circuit \\
\hline (17) & 118.9 & $126(9)$ & $T C-118.9-3 B-I R-3$ & PS-118.9-GR-IR-1 safety circuit \\
\hline$\langle 17\rangle$ & 118.9 & $92(9)$ & $T C-118 \cdot 9-4 C-I R-8$ & PS-118.9-GR-IR-1 safety circuit \\
\hline (17) & 118.9 & $138(g)$ & TC $-118.9-3 D-I R-8$ & PS-118.9-GR-IR-1 safety circuit \\
\hline (18) & 139.9 & $129(\mathrm{e})$ & $T C-139.9-6 \mathrm{~F}-\mathrm{S}-\mathrm{C}$ & PS-139.9-SH-1(b) protective circuit \\
\hline (18) & 139.9 & $128(e)$ & TC-139.9-6A-S-C & PS-139.9-SH-1 (b) protective circuit \\
\hline (18) & 139.9 & $131(\mathrm{e})$ & $T C-139.9-1 \mathrm{~A}-\mathrm{S}-\mathrm{C}$ & PS-139.9-SH-1 (b) protective circuit \\
\hline (18) & 139.9 & $13 D^{(e)}$ & TC-139.9-1F-S-C & PS-139.9-SH-1 (b) protective circuit \\
\hline (18) & 139.9 & 87 & $T C-139.9-6 E-I R-2$ & \\
\hline (18) & 139.9 & 73 & $T C-139.9-5 A-I R-3$ & Failed in MT-3.06 \\
\hline (18) & 139.9 & 66 & $T C-139.9-1 B-I R-4$ & \\
\hline (18) & 139.9 & 71 & TC-139.9-2F-IR-1 & \\
\hline- & 148.2 & & $\mathrm{TC}-148.2-4 \mathrm{E}-\mathrm{SP}-1$ & \\
\hline-- & 148.2 & & $T C-148.2-3 B-S P-3$ & \\
\hline-- & 148.2 & & $T C-148.2-3 E-S P-4$ & \\
\hline- & 151.5 & 6 & TC-151.5-5D-IR-C & \\
\hline (19) & 156.0 & 79 & $T C-156.0-50-B$ & \\
\hline (19) & 156.0 & 70 & $\mathrm{TC}-156.0-3 \mathrm{~B}-\mathrm{B}$ & \\
\hline (20) & 168.7 & 7 & $\mathrm{TC}-168.7-0 \mathrm{~T}-1$ & \\
\hline$(20)$ & 168.7 & 27 & $\mathrm{TC}-168.7-0 \mathrm{~T}-2$ & \\
\hline (20) & 168.7 & 3 & IC-168.7-0T-3 & \\
\hline (20) & 168.7 & 8 & $\mathrm{TC}-168.7-0 \mathrm{~T}-4$ & \\
\hline
\end{tabular}


TABLE 1. (contd)

\begin{tabular}{|c|c|c|c|c|}
\hline \multicolumn{2}{|c|}{$\begin{array}{l}\text { Instrument } \\
\text { Level }\end{array}$} & \multirow{2}{*}{$\begin{array}{l}\text { DACS } \\
\text { Channel } \\
\text { Number } \\
\end{array}$} & \multirow[b]{2}{*}{ Sensor Name } & \multirow[b]{2}{*}{ Notes } \\
\hline Tord) & New & & & \\
\hline (21) & 187.4 & 22 & TC-187.4-HT-1 & \\
\hline (21) & 187.4 & CRNL & TC-187.4-HT-2 & \\
\hline (21) & 187.4 & CRNL & TC-187.4-HT-3 & \\
\hline (21) & 187.4 & CRNL & TC-187.4-HT-4 & \\
\hline- & $H M(h)$ & 173 & $\mathrm{PT}-\mathrm{M} 1-3 \mathrm{~B}$ & Pressure transducer \\
\hline _- & HM & 174 & PT-M1-4B & Pressure transducer \\
\hline-- & HM & 175 & PT-M1-2B & Pressure transducer \\
\hline -- & HM & 176 & PT-MI-3B & Pressure transducer \\
\hline -- & HM & 177 & PT-M1-4B & Pressure transducer \\
\hline -- & HM & 178 & PT-M1-5B & Pressure transducer \\
\hline -- & HM & 179 & PT-M1-2B & Pressure transducer \\
\hline-- & HM & 180 & PT-M1-3B & Pressure transducer \\
\hline -- & HM & 181 & PT-M1-4B & Pressure transducer \\
\hline _- & HM & 182 & PT-MI-5B & Pressure transducer \\
\hline-- & HM & 183 & PT-MI-3B & Pressure transducer \\
\hline -- & HM & 184 & PT-M1 - 4B & Pressure transducer \\
\hline -- & 139.9 & 169 & $L L-D-D P$ & Liquid level detector $(j)$ \\
\hline-- & $F L^{(i)}$ & 172 & LL-D-TDR & Liquid level detector $(j)$ \\
\hline$(4 A)$ & 25.5 & 167 & ND-25.5-6F-S-3 & Failed in MT-3.04 \\
\hline (4A) & 25.5 & 143 & ND $-25.5-1 \mathrm{~A}-\mathrm{S}-1$ & SPND \\
\hline$(8 \mathrm{~A})$ & 46.5 & 145 & $N D-46.5-6 A-S-2$ & SPND \\
\hline (8A) & 46.5 & 146 & ND $-46.5-1 F-S-4$ & SPND \\
\hline (11A) & 67.5 & 142 & $N D-67.5-6 F-S-3$ & Failed (reading high) \\
\hline (11A) & 67.5 & 148 & $N D-67.5-6 A-S-2$ & Failed in MT-3.1 \\
\hline (11A) & 67.5 & 151 & ND-67.5-1A-S-1 & SPND \\
\hline (11A) & 67.5 & 150 & ND $-67.5-1 F-S-4$ & SPND \\
\hline (12) & 69.0 & 163 & $N D-69.0-1 F-C$ & Failed (reading high) \\
\hline (13A) & 88.5 & 155 & ND $-88.5-6 \mathrm{~F}-\mathrm{S}-3$ & SPND \\
\hline (13A) & 88.5 & 154 & ND-88.5-6A-S-2 & SPND \\
\hline$(13 A)$ & 88.5 & 157 & ND $-88.5-1 A-S-1$ & Failed in MT-3 preconditioning \\
\hline (13A) & 88.5 & 156 & ND-88.5-1F-S-4 & Failed in MT-3.04 \\
\hline
\end{tabular}


TABLE 1. (contd)

\begin{tabular}{|c|c|c|c|c|}
\hline \multicolumn{2}{|c|}{$\begin{array}{c}\text { Instrument } \\
\text { Leve1 }\end{array}$} & \multirow{2}{*}{$\begin{array}{l}\text { DACS } \\
\text { Channe } 1 \\
\text { Number }\end{array}$} & \multirow[b]{2}{*}{ Sensor Name } & \multirow[b]{2}{*}{ Notes } \\
\hline (D1d) & New & & & \\
\hline (15A) & 109.5 & 160 & ND-109.5-6F-S-3 & SPND \\
\hline (15A) & 109.5 & 159 & $N D-109.5-6 A-S-2$ & SPND \\
\hline (15A) & 109.5 & 162 & ND-109.5-1A-S-1 & Failed (reading low) \\
\hline$(15 A)$ & 109.5 & 161 & ND-109.5-1F-S-4 & Failed (reading low) \\
\hline$(16)$ & 111.0 & 152 & $N D-111.0-1 F-C$ & SPND \\
\hline (17A) & 130.5 & 166 & ND-130.5-6F-S-3 & SPND \\
\hline (17A) & 130.5 & 165 & $N D-130.5-6 A-S-2$ & SPND \\
\hline$(17 \mathrm{~A})$ & 130.5 & 168 & $N D-130.5-1 A-S-1$ & SPND \\
\hline$(17 A)$ & 130.5 & 149 & ND-130.5-1F-S-4 & SPND \\
\hline$(18 \mathrm{~A})$ & 151.5 & 170 & ND $-151.5-6 F-S-3$ & Failed in MT-3 preconditioning \\
\hline$(18 A)$ & 151.5 & 171 & ND-151.5-1A-S-1 & SPND \\
\hline \multicolumn{2}{|c|}{$U-1 L(k)$} & 201 & SRCS-FR-LO-W & $\mathrm{FE}-4$ reflood rate low \\
\hline \multicolumn{2}{|c|}{$U-1 L$} & 202 & SRCS-FR-HI-GH & FE-3 reflood rate high \\
\hline \multirow{2}{*}{\multicolumn{2}{|c|}{$\begin{array}{l}U-1 L \\
U-2 L(1)\end{array}$}} & 203 & STBY-FL-OW & FE-9 reflood emergency \\
\hline & & 204 & U2LP-PR-ES-S-1 & PDT-90 loop back pressure \\
\hline \multicolumn{2}{|c|}{$U-2 L$} & 205 & U2LP-TA-PS-DR-1 & PDT-90 in/out pressure drop \\
\hline \multicolumn{2}{|c|}{$U-1 L$} & 206 & SRCS-S-TC-IN-1 & $T E-2$ in steam temperature \\
\hline \multicolumn{2}{|c|}{$U-1 \mathrm{~L}$} & 207 & SRCS-S-TC-0T-1 & TE- 3 out steam temperature \\
\hline \multicolumn{2}{|c|}{$U-1 L$} & 208 & SRCS-S-PS-IN-1 & PT-5 in steam pressure \\
\hline \multicolumn{2}{|c|}{$U-1 L$} & 209 & SRCS-S-PS-0T-1 & PT- 6 out steam pressure \\
\hline \multicolumn{2}{|c|}{$U-1 L$} & 210 & SPCS-S-FR-1 & FY-6 steam flow rate (fajled) \\
\hline \multicolumn{2}{|c|}{$U-1 L$} & 211 & SRCS-S-FR-IN-1 & FV-1 in steam rate (failed) \\
\hline \multicolumn{2}{|c|}{$U-1 L$} & 212 & SRCS-S-FR-0T-1 & FI-2 out steam rate (failed) \\
\hline \multirow{2}{*}{\multicolumn{2}{|c|}{$U-1 L$}} & 213 & SRCS-TC-RF - LP-1 & TE-17 - Reflood loop temperature \\
\hline & & 214 & SRCS-TC - RF - TA-1 & TE-18 - Reflood assembly temperature \\
\hline \multirow{2}{*}{\multicolumn{2}{|c|}{$\begin{array}{l}U-1 L \\
U-2 L\end{array}$}} & 215 & U2LP-FL-OW-1 & FT-4D - water flow rate \\
\hline & & 216 & UNDEF I NED & \\
\hline \multicolumn{2}{|c|}{$U-1 L$} & 217 & SRCS-S-PS-0T-2 & PT-4 Outlet steam pressure \\
\hline \multicolumn{2}{|c|}{$U-1 \mathrm{~L}$} & 218 & ACUM-WT & Reflood weight \\
\hline \multirow{2}{*}{\multicolumn{2}{|c|}{$\begin{array}{l}U-2 L \\
11-2 L\end{array}$}} & 221 & U2LP-TC-IN-I & TE-78 Inlet water temperature \\
\hline & & 222 & U2LP-TC-0T-1 & TE-79 Outlet water temperature \\
\hline
\end{tabular}


TABLE 1. (contd)

\begin{tabular}{|c|c|c|c|c|}
\hline \multicolumn{2}{|c|}{$\begin{array}{c}\text { Instrument } \\
\text { Level }\end{array}$} & \multirow{2}{*}{$\begin{array}{l}\text { OACS } \\
\text { Channe1 } \\
\text { Number } \\
\end{array}$} & \multirow[b]{2}{*}{ Sensor Name } & \multirow[b]{2}{*}{ Notes } \\
\hline (01d) & $\mathrm{New}$ & & & \\
\hline \multicolumn{2}{|c|}{ U-1L } & 223 & SRCS-FR-HI-B & FE-3B Reflood flow high \\
\hline \multicolumn{2}{|c|}{$U-1 L$} & 224 & SRCS-FR-LO-B & FE-4B Reflood flow low \\
\hline \multicolumn{2}{|c|}{$U-1 L$} & 257 & SRC-S-OE-LT-A & LCS control switch \\
\hline \multicolumn{2}{|c|}{$\operatorname{NRU}(m)$} & 258 & SRC-RF-TR-IP & \multirow[t]{25}{*}{ LCS $(m)$ NRU trip switch } \\
\hline (1) & -0.2 & 289 & PS- $(-) 0.2-\operatorname{In}-1$ & \\
\hline (2) & 8.7 & 290 & PS-08.7-SH-1 & \\
\hline (3) & 13.9 & 291 & PS-13.9-SH-1 & \\
\hline (3) & 13.9 & 292 & PS-13.9-CR-2 & \\
\hline (4) & 19.2 & 293 & PS-19.2-SH-1 & \\
\hline (5) & 28.0 & 294 & PS-28.0-SH-1 & \\
\hline (6) & 34.9 & 295 & PS-34.9-SH-1 & \\
\hline (7) & 40.2 & 296 & PS-40.2-GR-IR-1 & \\
\hline (7) & 42.9 & 297 & PS-42.9-SH-1 & \\
\hline (8) & 42.9 & 298 & PS-42.9-SH-1 & \\
\hline (9) & 55.9 & 299 & PS-55.9-SH-1 & \\
\hline \multirow[t]{4}{*}{ (10) } & 55.9 & 300 & PS- 55.9-SH-1 & \\
\hline & 61.2 & 301 & PS-61.2-GR-IR-1 & \\
\hline & 69.0 & 302 & PS-69.0-CR-SR-1 & \\
\hline & 73.9 & 303 & PS-73.9-CR-IR-1 & \\
\hline \multirow[t]{2}{*}{ (13) } & 76.9 & 304 & PS-76.9-SH-1 & \\
\hline & 118.9 & 305 & PS-118.9-SH-2 & \\
\hline \multirow[t]{4}{*}{ (13) } & 76.9 & 306 & PS-CR-IR- 1 & \\
\hline & 78.9 & 307 & PS-78.9-CR-IR-1 & \\
\hline & 80.9 & 308 & PS-80.9-CR-IR-1 & \\
\hline & 83.9 & 309 & PS-83.9-CR-IR-1 & \\
\hline \multirow{5}{*}{$\begin{array}{l}(14) \\
(15)\end{array}$} & 90.0 & 310 & PS-90.0-CR-SP-1 & \\
\hline & 97.9 & 311 & PS-97.9-SH-1 & \\
\hline & $97.9(0)$ & 312 & PS-97.9-GR-1R-1 $(0)$ & \\
\hline & 97.9 & 313 & PS-97.9-GR-1R-2 & $103,53,45,24,2^{(0)}$ \\
\hline & 97.9 & 314 & PS-97.9-GR-C-1 & \\
\hline \multirow[t]{3}{*}{ (14) } & 97.9 & 315 & PS-97.9-CR-IR-1 & \\
\hline & 101.9 & 316 & PS-101.9-CR-IR-1 & \\
\hline & 104.9 & 317 & PS-104.9-CR-IR-1 & \\
\hline \multirow[t]{3}{*}{ (16) } & 111.0 & 318 & PS-111.0-CA-1 & \\
\hline & 118.9 & 319 & PS-118.9-SH-1 & \\
\hline & 118.9 & 320 & PS-118.9-SH-1 & \\
\hline (17) & 118.9 & 321 & PS-118.9-CR-IR-1 $(0)$ & $138,126,92,81(0)$ \\
\hline
\end{tabular}


TABLE 1. (contd)

\begin{tabular}{|c|c|c|c|c|}
\hline \multicolumn{2}{|c|}{$\begin{array}{c}\begin{array}{c}\text { Instrument } \\
\text { Leve }\end{array} \\
\end{array}$} & \multirow{2}{*}{$\begin{array}{l}\text { DACS } \\
\text { Channel } \\
\text { Number } \\
\end{array}$} & \multirow[b]{2}{*}{ Sensor Name } & \multirow[b]{2}{*}{ Notes } \\
\hline (01d) & $\mathrm{New}$ & & & \\
\hline \multirow[t]{3}{*}{ (18) } & 139.9 & 322 & PS-139.9-SH-1 & \\
\hline & 139.9 & 323 & PS-139.9-GR-IR-1 & \\
\hline & 148.2 & 324 & PS-148.2-CR-SR-1 & \\
\hline \multirow{3}{*}{ (20) } & 155.7 & 324 & PS-155.7-CR-8-1 & \\
\hline & 168.7 & 326 & PS-168.7-0T-1 & \\
\hline & 75.9 & 327 & PS-76.9-GR-IR-1 & \\
\hline
\end{tabular}

(a) Distance above top of the tie plate, e.g., $-0.2 \mathrm{in.}, 8.7 \mathrm{in.}$, and $13.9 \mathrm{in}$.

(b) Failed during preconditioning.

(c) These sensors are located at a different axial location than in previous tests.

(d) Pseudo sensor (DACS No. 327) PS-76.9-GR-IR-1 is composed of DACS Nos. 1D1, 52,50 , and 67.

(e) Protective trip sensors and pseudo sensors.

(f) Safety pseudo sensor (DACS No. 312) PS-97.9-GR-IR-1 is composed of DACS Nos. $103,25,45,24$, and 2 .

(g) Safety pseudo sensor (DACS No. 321) PS-118.9-GR-IR-1 is composed of DACS Nos. $81,126,92$, and 138 .

(h) Head-mounted pressure transducers that were connected to fuel rods with highpressure tubes.

(i) Full-length TDR liquid level detector.

(j) Sampled coolant and orifice pressure drop.

(k) Located in the U-1 loop of the NRU (steam- and reflood-cooled system).

(1) Located in the U-2 loop of the NRU (water-cooled system).

(m) NRU safety trip circuit indicator.

(n) Safety trip circuit pseudo sensors and "averaged" DACS numbers.

(o) Protective trip sensors.

required power distribution. (5) NRU reactor fuel operations reloaded specified $(6)$ fuel as required to assure less than a $5 \%$ radial power skew across the L-24 position.

\section{EXPERIMENT OPERATIONS}

The primary objective of the MT-4 experiment was to evaluate cladding ballooning and rupture during adiabatic heatup in the range from 1033 to $1200 \mathrm{~K}$ $\left(1400\right.$ to $\left.1700^{\circ} \mathrm{F}\right)$. To meet that objective, the unirradiated test fuel rods were first preconditioned. 
After installation, the test rod pressure system was purged, sampled, and maintained at relatively low pressure--0.21 $\mathrm{MPa}(30 \mathrm{psia})$. Two approaches to full-power NRU operation and two conditional reactor trips assured fuel pellet cracking and good fuel/cladding mechanical contact. This preconditioning operation provided up to 1 equivalent-full-power-hour (EFPH) of NRU irradiation with operating conditions similar to those of previous experiments. The operating conditions are summarized in Table 2.

After preconditioning operations were completed and the L-24 piping was connected to the U-1 loop for steam supply and the reflood system, reflood system calibrations began (see Table 2). Reflood calibrations focused on the mode of operation used in the MT-4 transient, preset reflood delay time and flow rates, and computer-controlled flow after the transient. Initial operations checked out the prefill and drain sequence that delivered reflood water to the bottom of the fuel column. Constant reflood flow rates were calibrated from 0 to $0.0508 \mathrm{~m} / \mathrm{s}(2.0 \mathrm{in.} / \mathrm{s})$, at $0.0965 \mathrm{~m} / \mathrm{s}(3.8 \mathrm{in.} / \mathrm{s})$, and at $0.1219 \mathrm{~m} / \mathrm{s}$ $(4.8 \mathrm{in.} / \mathrm{s})$. Pretransient power coupling measurements were made using adiabatic heatup and standard calorimetric techniques with steam cooling at a power level of $\sim 6$ to $8 \mathrm{MW}$. During these measurements, the protective trip circuitry was also verified using a fuel cladding trip set point at $\sim 922 \mathrm{~K}$ $\left(1200^{\circ} \mathrm{F}\right)$ to terminate the calibration tests.

When it was confirmed that the test assembly instrumentation and reflood loop control system (LCS) were ready, the 12 test rods were pressurized to $\sim 4.62 \mathrm{MPa}$ (670 psia) using the pressure system mounted on the NRU reactor head. The pressure tubes were subsequently sealed and independently monitored during the remainder of the experiment. MT-4 transient operation repeated the operating conditions used in TH-1.16. (4) After steam flow stopped, reflood flow was delayed for $\sim 57 \mathrm{~s}$. Reflood $\mathrm{flow}$ at a rate of $\sim 0.0965 \mathrm{~m} / \mathrm{s}$ turned the peak cladding temperature $(1200 \mathrm{~K})$ around at $\sim 70 \mathrm{~s}$ into the transient.

After the peak fuel cladding temperature was reached, reflood flow was stopped briefly and then re-established (with computer control) at $\sim 0.00508 \mathrm{~m} / \mathrm{s}$ $(0.2 \mathrm{in.} / \mathrm{s})$ to stabilize the cladding temperature at $\sim 1089 \mathrm{~K}\left(1500^{\circ} \mathrm{F}\right)$ for postLOCA heat transfer coefficient measurements (2 to 3 min). Reflood flow was again reduced to establish a stable cladding temperature at $\sim 1300 \mathrm{~K}\left(1700^{\circ} \mathrm{F}\right)$ and to enable similar heat transfer coefficient measurements ( 2 to $3 \mathrm{~min}$ ). The experiment was terminated with a conditional NRU reactor trip and test assembly quench using reflood cooling. 
TABLE 2. MT-4 Operating Conditions

\begin{tabular}{|c|c|c|c|c|}
\hline & & Calibrations & & \\
\hline Parameter & Preconditioning & Steam, Reflood, water & Pretranslent & Transient ${ }^{(B)}$ \\
\hline Reactor power, MW & 127 & 0 to 8 & 8 & B \\
\hline Coolant & U-2 water & U-1 steam & $v-1$ staen $(a)$ & Reflood vater \\
\hline $\begin{array}{l}\text { Infet temperature, } \\
K\left({ }^{\circ} F\right\rangle\end{array}$ & $518\langle 472\rangle$ & $435(325)$ & $435(325)$ & NA \\
\hline $\begin{array}{l}\text { Coolant flom, } \\
\mathrm{kg} / \mathrm{s}(\mathrm{lbm} / \mathrm{h})\end{array}$ & $\begin{array}{l}0 \text { to } 16.3 \\
(0 \text { to } 129,400)\end{array}$ & $0.378(3000)$ & $0.378(3000)$ & 0 \\
\hline Reflood delay, s & NA & 0 to 51 & NA & 51 \\
\hline $\begin{array}{l}\text { Reflood rate, } \\
m / s\left(i n_{-} / s\right)\end{array}$ & NA & $\begin{array}{r}0 \text { to } 0.1219 \\
(0 \text { to } 4.8)\end{array}$ & NA & $\begin{array}{l}0 \text { to } 0.0965 \\
10 \text { to } 3.81\end{array}$ \\
\hline $\begin{array}{l}\text { Reflood temperature, } \\
K\left({ }^{\circ} \mathrm{F}\right)\end{array}$ & NA & $311(100)$ & NA & $311(100)$ \\
\hline $\begin{array}{l}\text { Peak cladding } \\
\text { temperature, } k\left({ }^{\circ} \mathrm{F}\right)\end{array}$ & $700(800)$ & $444(340)$ & $728(850)$ & $1200(1700)$ \\
\hline $\begin{array}{l}\text { Reactor trip } \\
\text { criter la }(b)\end{array}$ & Safety & $\begin{array}{l}\text { Protective } \\
\text { and safety }\end{array}$ & $\begin{array}{l}\text { Protectlve } \\
\text { and safety }\end{array}$ & $\begin{array}{l}\text { Protective } \\
\text { and safety }\end{array}$ \\
\hline
\end{tabular}

(a) Transient initiated by termination of steam tlaw.

(b) See Table 3 for trip set points and operating limits. 
PROTECTIVE AND SAFETY TRIPS

Previous transient tests qualified the protective trip set points, overshoot, and the protective trip circuitry. Protective trips were designed to limit fuel temperatures to that range compatible with the experiment objectives and operations.

Protective trip logic limited test fuel temperatures in several ways: maximum allowable fuel cladding temperature, maximum and minimum allowable heatup rates, and time limitations for both fuel cladding temperatures and coolant reflooding to preselected levels. These protective trip options and their set points were selected by the experimenter at various times during the course of the tests. The protective trip signal was designed for several operating condition criteria, and the LCS and NRU reactor controls always responded the same: 1) the reactor was conditionally tripped and 2) emergency reflood flow was initiated.

The protective trip logic was based on sensor sampling as frequently as 10 times per second. The safety trip logic and circuitry were nearly all the same as designed for previous experiments. (3) The only revision was the removal of the minimum reflood flow rate limit to permit the use of zero and very low reflood flow rates during post-transient operation. During that period, temperature stabilization was provided by computer-controlled reflood flow rates that included zero flow intervals. Safety trip set points are summarized in Tables 3 and 4 . Protective trip set points were selected and activated by the experimenter at various times during the experiment. Both protective and safety trips utilized the same hard-wired circuitry to achieve an NRU reactor trip. Each trip condition had its own independent program logic; thus, safety trips were not influenced by any change in protective trips. 
TABLE 3. Pretransient and Transient Safety Trip Set Points(a)

\begin{tabular}{|c|c|c|c|c|}
\hline Parameter & Location & Use & $\begin{array}{c}\text { Operating } \\
\text { Limits }\end{array}$ & $\begin{array}{l}\text { Safety Trip } \\
\text { Set Point }\end{array}$ \\
\hline Hanger tube temperature - high & LCS & $\begin{array}{l}\text { Pretransient } \\
\text { and transient }\end{array}$ & $691 \mathrm{~K}\left(785^{\circ} \mathrm{F}\right)$ & $839 \mathrm{~K}\left(1050^{\circ} \mathrm{F}\right)$ \\
\hline Outlet pipe temperature - high & LCS & $\begin{array}{l}\text { Pretransient } \\
\text { and transient }\end{array}$ & $672 \mathrm{~K}\left(750^{\circ} \mathrm{F}\right)$ & $700 \mathrm{~K}\left(800^{\circ} \mathrm{F}\right)$ \\
\hline $\begin{array}{l}\text { Fuel cladding temperature - high(a) } \\
\text { Level (13) } 76.9305 \text { PS- } 76.9-G R-I R-1 \\
\text { Level (15) } 97.9308 \text { PS-97.9-GR-IR-1 } \\
\text { Leve1 (17) } 118.9315 \text { PS-118.9-GR-IR-1 }\end{array}$ & $\begin{array}{l}\text { OACS } \\
\text { DACS } \\
\text { DACS }\end{array}$ & Transient & $\begin{array}{l}1305 \mathrm{~K}\left(1890^{\circ} \mathrm{F}\right) \\
1305 \mathrm{~K}\left(1890^{\circ} \mathrm{F}\right) \\
1278 \mathrm{~K}\left(1840^{\circ} \mathrm{F}\right)\end{array}$ & $\begin{array}{l}1361 \mathrm{~K}\left(1990^{\circ} \mathrm{F}\right) \\
1361 \mathrm{~K}\left(1990^{\circ} \mathrm{F}\right) \\
1333 \mathrm{~K}\left(1940^{\circ} \mathrm{F}\right)\end{array}$ \\
\hline Low steam flow trip & LCS & $\begin{array}{l}\text { Pretransient } \\
(30001 \mathrm{bm} / \mathrm{h})\end{array}$ & $\begin{array}{l}0.378 \mathrm{~kg} / \mathrm{s} \\
(2200 \mathrm{lbm} / \mathrm{h})\end{array}$ & $0.277 \mathrm{~kg} / \mathrm{s}$ \\
\hline Accumulator inventory - low & $\operatorname{LCS}$ & Transient & $22.7 \mathrm{~kg}(501 \mathrm{bm})$ & $11.3 \mathrm{~kg}(251 \mathrm{bm})$ \\
\hline
\end{tabular}

(a) Standard trip set point criteria; see Table 4 for nonfunctional TC criteria. 
TABLE 4. Standard and Defaulted Safety Trip Set Point Criteria for Operating and Nonfunctional Fuel Cladding Thermocouples

\begin{tabular}{|c|c|c|c|c|}
\hline \multirow[b]{4}{*}{ Operating Conditions } & \multirow{4}{*}{$\begin{array}{c}\text { Criteria and } \\
\text { Safety Margins } \\
\text { (SM) }\end{array}$} & $\begin{array}{c}\text { Sensors at } \\
\text { Level (17) } 118.9\end{array}$ & $\begin{array}{c}\text { Sensors at } \\
\text { Level (15) } 97.9 \\
\end{array}$ & \multirow[t]{2}{*}{$\begin{array}{c}\text { Sensors at } \\
\text { Level (13) } 76.9 \\
\end{array}$} \\
\hline & & \multicolumn{2}{|c|}{ DACS Thermocouple Kumbers } & \\
\hline & & $\begin{array}{c}\text { 315, PS-118.9-GR-IR-1 (a) } \\
(81,126,92,138) \\
\end{array}$ & $\begin{array}{c}308, \text { PS-97.9-GR-IR-1 } \\
(103,25,45,24,2) \\
\end{array}$ & $\begin{array}{c}305, \text { PS-76.9-GR-IR-1 } \\
(101,52,50,67) \\
\end{array}$ \\
\hline & & \multicolumn{3}{|c|}{ Safety Trip set Point Termeratures, $\bar{k}\left({ }^{\circ} \mathrm{F}\right)$} \\
\hline $\begin{array}{l}2 \leq \text { Number of operating } \\
\text { TCS on each of } \\
\text { Levels } 76.9,97.9 \text {, } \\
\text { and } 118.9\end{array}$ & $\begin{array}{c}\text { Standard } \\
\text { SM }=56 \mathrm{~K}\left(100^{\circ} \mathrm{F}\right)\end{array}$ & $1333(1940)$ & $1361(1990)$ & $1361(1990)$ \\
\hline $\begin{array}{c}2 \leq \text { Nmber of operating } \\
\text { TCs on each of } \\
\text { Levels } 97.9 \text { and } \\
118.9\end{array}$ & $\begin{array}{c}\text { Standard } \\
S M=56 \mathrm{~K}\left(100^{\circ} \mathrm{F}\right)\end{array}$ & $1333(1940)$ & $1361(1990)$ & \\
\hline $\begin{array}{c}2 \leq \text { Number of operating } \\
\text { TCs on each of } \\
\text { levels } 97.9 \text { and } \\
118.9 \text { or Levels } \\
76.9 \text { and } 118.9\end{array}$ & $\begin{array}{c}\text { Alternate } \\
S M=84 \mathrm{~K}\left(150^{\circ} \mathrm{F}\right)\end{array}$ & $1305(1890)$ & $1333(1940)$ & $1333(1940)$ \\
\hline $\begin{array}{l}2 \leq \text { Nunber of operating } \\
\text { TCs on only Level } \\
97.9,118.9 \text {, or } \\
76.9\end{array}$ & $\begin{array}{c}\text { Fall back } \\
S M=111 \mathrm{~K}\left(200^{\circ} \mathrm{F}\right)\end{array}$ & $1277(1840)$ & $1305(1890)$ & $1305(1890)$ \\
\hline
\end{tabular}

(a) These pseudo sensor data (PSD) are the calculated time-average of the follawing DACS sensor-numbered data: 81, 126, 92, 138. 


\section{SAFETY HAZARDS REVIEW}

The MT-4 experiment was similar to previous experiments (TH-1.16, MT-2, and $M T-3)$. Both the MT-4 operating conditions and the expected fuel failure mechanisms had been tested in previous experiments.

The same operating conditions that were used in $T H-1.16$ and MT-3 were used in MT-4. Fuel preconditioning was produced by up to $1 \mathrm{EFPH}$ in the NRU reactor. The simulated LOCA transient was produced by a reflood delay of $\sim 57 \mathrm{~s}$ followed by reflood coolant flow at $\sim 0.0965 \mathrm{~m} / \mathrm{s}(3.8 \mathrm{in.} / \mathrm{s})$ (same as in TH-1.16). For MT-4, however, the 12 test rods were pressurized just prior to the transient that caused ballooning and rupture, which precluded premature ballooning and rupture during earlier transients. After the transient, fuel cladding high temperatures stabilized and the procedures and operating ranges established in MT-2 and MT-3 were used. Computer-controlled reflood flow enabled thermalhydraulic data to be collected on the deformed test assembly during oscillatory operating conditions.

The 12 test rods were expected to balloon and rupture $\sim 60$ to 80 s into the transient when the fuel cladding reached $\sim 1144 \mathrm{~K}\left(1600^{\circ} \mathrm{F}\right)$. The neutronic envi ronment and the fuel rod internal pressure were carefully monitored in $V_{i T-4 .}$ The MT-4 experiment was the first experiment to use pressure transducers located outside the NRU reactor to provide more reliable rupture times. The 12 pressure transducers were mounted above the reactor head but were connected to the test rods with high-pressure Type 321 stainless steel tubing. (a) The lowest yield strength pressure rating of the (weakest) pressure system component outside the reactor (high-pressure tubing) that communicated with fission gas in the test fuel rods was $106.9 \mathrm{MPa}(15,500 \mathrm{psi})$ at a temperature of $700 \mathrm{~K}$ $\left(800^{\circ} \mathrm{F}\right)$. This compares with a maximum credible hoop stress of $15.03 \mathrm{MPa}(2180$ psi) due to the fission gas pressure at the time of fuel cladding rupture. During transient operations, the valves on these high-pressure tubes were sealed to preclude fission gas leakage.

Other safety analysis reports $(2,7)$ are valid for the MT-4 experiment because the operating conditions, expected fuel failure mechanisms, and failure effects are within the envelope of cases analyzed there and reported for subsequent experiments.

(a) $1.65 \mathrm{~mm}(0.065 \mathrm{in.})$ outside diameter and $0.635 \mathrm{~mm}(0.025 \mathrm{in.})$ inside diameter. 



\section{MT-4 OPERATING PROCEDURE}

PRECONDITIONING OPERATIONS

Test Configuration

1. Install MT-4 test assembly in L-24 NRU reactor position.

2. Purge test fuel rod pressure system and collect helium samples before preconditioning.

3. Install traveling fission chamber in $\mathrm{J}-22$ reactor position.

4. Connect U-2 loop to L-24 test position.

5. Pressure test the test train head seal.

Loop System Preparations

1. Fill and electrically preheat the U-2 loop.

2. Provide water chemistry as shown in Table 5.

3. Calibrate loop instruments (see Table 6).

4. Confirm trip circuit operability.

5. Implement trip set points (see Table 7).

TABLE 5. Water Chemistry Requirements

\begin{tabular}{|c|c|c|}
\hline Requi rement & Applicability & Recommended Limits \\
\hline $\begin{array}{l}\text { Deionized } \\
\text { supply }\end{array}$ & $\begin{array}{l}\text { Preconditioning } \\
\text { water coolant }\end{array}$ & $\leq 1 \times 10^{-5}$ Mho \\
\hline $\begin{array}{l}\text { Impurity } \\
\text { concentrations }\end{array}$ & $\begin{array}{l}\text { Halides } \\
\text { Oxygen } \\
\text { All other elements }\end{array}$ & $\begin{array}{l}\leq 1 \mathrm{ppm} \\
\leq 100 \mathrm{ppm} \\
\leq 100 \mathrm{ppm}\end{array}$ \\
\hline
\end{tabular}


TABLE 6. U-2 Loop Instrument Calibration

\begin{tabular}{|c|c|c|c|c|}
\hline Sensor & Loop Parameter & DACS & Instrument Range & $\begin{array}{l}\text { Acceptable } \\
\text { Accuracy }\end{array}$ \\
\hline TE-78 & Inlet temperature & 221 & $\begin{array}{l}311 \text { to } 533 \mathrm{~K} \\
\left(100 \text { to } 500^{\circ} \mathrm{F}\right)\end{array}$ & $\begin{array}{l} \pm 1 \mathrm{~K} \\
\left( \pm 2^{\circ} \mathrm{F}\right)\end{array}$ \\
\hline TE-79 & Outlet temperature & 222 & $\begin{array}{l}323 \text { to } 589 \mathrm{~K} \\
\left(122 \text { to } 600^{\circ} \mathrm{F}\right)\end{array}$ & $\begin{array}{l} \pm 1 K \\
\left( \pm 2^{\circ} \mathrm{F}\right)\end{array}$ \\
\hline FT-40 & Flow & 215 & $\begin{array}{l}3.9^{(a)} \text { to } 19.3^{(b)} \mathrm{kg} / \mathrm{s} \\
(36,400 \text { to } 131,400 \mathrm{lbm} / \mathrm{h})\end{array}$ & $\begin{array}{l} \pm 0.4 \mathrm{~kg} / \mathrm{s} \\
( \pm 2000 \mathrm{lbm} / \mathrm{h}\rangle\end{array}$ \\
\hline PDT-90 & Outlet pressure & 204 & $\begin{array}{l}5.52 \text { to } 8.96 \mathrm{MPa} \\
(800 \text { to } 1300 \mathrm{psia})\end{array}$ & $\begin{array}{l} \pm 0.3 \mathrm{MPa} \\
( \pm 50 \mathrm{psia})\end{array}$ \\
\hline PDT-90 & $\begin{array}{l}\text { Test assembly } \\
\text { pressure drop }\end{array}$ & 205 & $\begin{array}{l}0.021 \text { to } 0.172 \mathrm{MPa} \\
\text { (3 to } 25 \mathrm{psi})\end{array}$ & $\begin{array}{l} \pm 0.002 \mathrm{MPa} \\
( \pm 0.3 \mathrm{psi})\end{array}$ \\
\hline
\end{tabular}

(a) At a temperature of $589 \mathrm{~K}\left(600^{\circ} \mathrm{F}\right)$.

(b) At a temperature of $394 \mathrm{~K}\left(250^{\circ} \mathrm{F}\right)$.

TABLE 7. Preconditioning Safety Trip Set Pounts

\begin{tabular}{|c|c|c|}
\hline Parameter & $\begin{array}{c}\text { Nominal } \\
\text { Operating Limits } \\
\end{array}$ & Trip Set Point \\
\hline Outlet coolant temperature & $552 \mathrm{~K}\left(534^{\circ} \mathrm{F}\right)$ & $561 \mathrm{~K}\left(550^{\circ} \mathrm{F}\right)$ \\
\hline $\begin{array}{l}\text { Pump subcooling } \\
\text { temperature--low }\end{array}$ & $338 \mathrm{~K}\left(150^{\circ} \mathrm{F}\right)$ & $283 \mathrm{~K}\left(50^{\circ} \mathrm{F}\right)$ \\
\hline Coolant flow--low & $\begin{array}{l}15.5 \mathrm{~kg} / \mathrm{s}(\mathrm{a}) \\
(123,000 \mathrm{bm} / \mathrm{h})\end{array}$ & $\begin{array}{l}13.4 \mathrm{~kg} / \mathrm{s}(\mathrm{a}) \\
(106,300 \mathrm{lbm} / \mathrm{h})\end{array}$ \\
\hline Surge tank level--low & $\operatorname{TBD}(b)$ & $30 \%$ \\
\hline Surge tank pressure--high & $\mathrm{TBD}(\mathrm{b})$ & $8.90 \mathrm{MPa}(1275 \mathrm{psig})$ \\
\hline
\end{tabular}

(a) At a temperature of $518 \mathrm{~K}\left(472^{\circ} \mathrm{F}\right)$.

(b) To be determined by CRNL. 
NEU Reactor Preparations

1. Load NRU reactor fuel assemblies and absorber assemblies as requi red. (6)

2. Adjust the neutron detector scatter plug as required.

3. Establish mean power trip set points as approved.

4. Confirm that all trip set points are activated, and report to the experiment director when ready for operation.

DACS Computer Preparations

1. Load labeled, certified disks and mount a labeled, certified tape on the tape drive.

2. Start a dummy test and set the DACS mode to idle.

3. Set the steady-state scan rate at $1 \mathrm{~s}$.

4. Set the immediate display scan rate at $4 \mathrm{~s}$.

5. Set the graphic display scan rate at $5 \mathrm{~s}$.

6. Format the steady-state immediate display.

7. Identify and remove failed sensors from both displays.

8. Verify trip circuit operability.

9. Set Keithley amplifiers to the 1.0 scale and verify that SPND coefficients are correct.

Preconditioning Operating Procedure (Total time at full NRU reactor power is to be limited to 1.0 EFPH.)

1. End the dummy test, and start a new test on the DACS.

2. Start the loop using sparge pumps to provide initial flow $[6.3 \mathrm{~kg} / \mathrm{s}$ $(129,400 \mathrm{lbm} / \mathrm{h})]$ and initial pressurization $[8.62 \mathrm{MPa}(1250 \mathrm{psia})]$.

3. Change the DACS mode to steady-state and turn on the video tape recorder. 
4. Adjust the inlet temperature to $518 \pm 3 \mathrm{~K}\left(472 \pm 5^{\circ} \mathrm{F}\right)$.

5. Print the DACS sensor status report and REDACE print-out for review for $\Delta T$ heat loss check.

6. Increase the reactor power to $63.5 \mathrm{MW}$; request REDACE print-out.

7. Perform a power calibration using a REDACE print-out and DACS data (loop flow rate times test assembly $\triangle T$ ).

8. Print the DACS sensor status report and REDACE print-out for review.

9. Increase the reactor power to $127 \mathrm{MW} \pm 5 \%$.

10. Print the DACS sensor status report and the REDACE print-out.

11. Perform a power calibration, including neutron flux data records (unless done at low power) using the traveling fission chamber in the J-22 reactor position.

12. Conditionally trip the reactor.

13. Repeat Steps 3 and 5.

14. Increase the reactor to full power $(127 \mathrm{MW} \pm 5 \%)$, and maintain test assembly in?et temperature at $518 \pm 3 \mathrm{~K}\left(472 \pm 5^{\circ} \mathrm{F}\right)$.

15. Print the DACS and REDACE sensor status reports.

16. Recheck power calibrations and traveling fission chamber data.

17. Conditionally trip the reactor.

18. Make a hard copy of the CRT immediate and graphic displays showing the hottest centerline TCs. Change DACS mode to idle. Make a tape copy when ending the test; make a disk image copy; and make a historical request for all the data required to run the transient test.

19. Shut down the loop facilities to prepare for piping rearrangement. Return DACS to steady-state, and scan the sensors once per minute until reflood flow tests are initiated.

20. Place one copy of all data records collected in system log and have data coordinator sign. 
REFLOOD CALIBRATION, PRETRANSIENT, AND TRANSIENT OPERATIONS

Reflood calibration, pretransient, and transient operations are discussed in this section. MT-4 operating conditions are summarized in Table 8.

Test Configuration

1. MT-4 assembly installed in L-24 NRU reactor position.

2. Fuel rod pressure system connected to the MT-4 test assembly.

3. Traveling fission chamber installed in $\mathrm{J}-22$ reactor position.

4. Reflood loop connected to the L-24 NRU reactor position.

5. U-1 steam supply connected to the reflood loop.

6. NRU reactor fuel and absorber assemblies loaded as required.(6)

Loop System Preparations

1. Start up the U-1 loop.

2. Insure that U-2 makeup tanks (which supply water to the U-1 ioop) are full.

3. Preheat the steam/reflood loop to $408 \mathrm{~K}\left(275^{\circ} \mathrm{F}\right)$.

4. Fill reflood accumulators at $311 \pm 6 \mathrm{~K}\left(100 \pm 10^{\circ} \mathrm{F}\right)$. Check water temperature in the three accumulators.

5. Verify that the nitrogen supply for accumulator pressurization is adequate.

6. Calibrate loop instruments as shown in Table 9. Enter conversion factors and units in the test log.

7. Implement the safety trip set points as shown in Tables 10 and 11 for the pretransient and transient phases of the experiment. The safety pseudo sensors used by the DACS to represent the high cladding temperature trip set points and sensors are identified on Table 12. 
TABLE 8. MT-4 Operating Conditions

\begin{tabular}{|c|c|c|c|c|c|}
\hline Parameter & \multicolumn{2}{|l|}{$\begin{array}{l}\text { Calibrations } \\
\text { Steam, Reflood, }\end{array}$} & Adiabatic Test & Pretransient & Transient \\
\hline Reactor power, MW & 0 to 8 & & 6 to 8 & 6 to 8 & 6 to 8 \\
\hline Coolant & U-I steam & U-2 water & U-1 steam $(a)$ & $U-1$ steam $(a)$ & Reflood water \\
\hline $\begin{array}{l}\text { Inlet temperature, } \\
\mathrm{K}\left({ }^{\circ} \mathrm{F}\right)\end{array}$ & $435(325)$ & $311(100)$ & $435(325)$ & $435(325)$ & NA \\
\hline $\begin{array}{l}\text { Coolant flow, } \\
\mathrm{kg} / \mathrm{s}(1 \mathrm{bm} / \mathrm{h})\end{array}$ & $\begin{array}{l}0.378 \\
(3000)\end{array}$ & $\begin{array}{l}0.441 \\
(3500)\end{array}$ & $0.378(3000)$ & $0.378(3000)$ & 0 \\
\hline Reflood delay, s & 0 to 51 & & NA & NA & 51 \\
\hline $\begin{array}{l}\text { Reflood rate, } \\
\text { m/s (in./s) }\end{array}$ & $\begin{array}{l}0 \text { to } 0.1219 \\
(0 \text { to } 4.8)\end{array}$ & & NA & NA & $\begin{array}{l}0 \text { to } 0.0965 \\
(0 \text { to } 3.8)\end{array}$ \\
\hline $\begin{array}{l}\text { Reflood temperature, } \\
K\left({ }^{\circ} \mathrm{F}\right)\end{array}$ & $311(100)$ & & NA & NA & $311(100)$ \\
\hline $\begin{array}{l}\text { Peak cladding } \\
\text { temperature, } k\left({ }^{\circ} \mathrm{F}\right)\end{array}$ & $444(340)$ & & $853(1075)$ & $728(850)$ & $1200(1700)$ \\
\hline $\begin{array}{l}\text { Reactor trjp } \\
\text { criteria }\end{array}$ & $\begin{array}{l}\text { Protective } \\
\text { and safety }\end{array}$ & & $\begin{array}{l}\text { Protective }(c) \\
\text { and safety }\end{array}$ & $\begin{array}{l}\text { Protective } \\
\text { and safety }\end{array}$ & $\begin{array}{l}\text { Protective } \\
\text { and safety }\end{array}$ \\
\hline $\begin{array}{l}\text { (a) Transient initiat } \\
\text { (b) See Table } 3 \text { for } \\
\text { (c) Protective trip }\end{array}$ & $\begin{array}{l}\text { by termination of } s \\
\text { pet points and ope } \\
\text { point was } 825 \mathrm{~K}(102\end{array}$ & $\begin{array}{l}\text { team flow. } \\
\text { rating } 1 \mathrm{imi} \\
5^{\circ} \mathrm{F} \text { ) for ad }\end{array}$ & ts. & & \\
\hline
\end{tabular}


TABLE 9. Steam/Reflood System Calibration (CRNL)

\begin{tabular}{|c|c|c|c|c|}
\hline Sensor & Parameter & DACS & Instrument Range & $\begin{array}{l}\text { Acceptable } \\
\text { Accuracy }\end{array}$ \\
\hline TE-2 & Inlet coolant temperature & 206 & $\begin{array}{l}394 \text { to } 700 \mathrm{~K} \\
\left(250 \text { to } 300^{\circ} \mathrm{F}\right)\end{array}$ & $\begin{array}{l} \pm 1 K \\
( \pm 2 F)\end{array}$ \\
\hline TE-3 & Outlet coolant temperature & 207 & $\begin{array}{l}422 \text { to } 973 \mathrm{~K} \\
\left(300 \text { to } 1300^{\circ} \mathrm{F}\right)\end{array}$ & $\begin{array}{l} \pm 6 K \\
\left( \pm 10^{\circ} \mathrm{F}\right)\end{array}$ \\
\hline$F Y-6$ & $\begin{array}{l}\text { Steam flow rate, reflood } \\
\text { boiling }\end{array}$ & 210 & $\begin{array}{l}0 \text { to } 0.378 \mathrm{~kg} / \mathrm{s} \\
(0 \text { to } 3000 \mathrm{lbm} / \mathrm{h})\end{array}$ & $\begin{array}{l} \pm 0.014 \mathrm{~kg} / \mathrm{s} \\
( \pm 100 \mathrm{lbm} / \mathrm{h})\end{array}$ \\
\hline$F V-1$ & $\begin{array}{l}\text { Inlet steam system control } \\
\text { flow rate }\end{array}$ & 211 & $\begin{array}{l}0 \text { to } 0.378 \mathrm{~kg} / \mathrm{s} \\
(0 \text { to } 3000 \mathrm{lbm} / \mathrm{h})\end{array}$ & $\begin{array}{l} \pm 0.014 \mathrm{~kg} / \mathrm{s} \\
( \pm 100 \mathrm{lbm} / \mathrm{h})\end{array}$ \\
\hline FI -2 & $\begin{array}{l}\text { Outlet steam system } \\
\text { control flow rate }\end{array}$ & 212 & $\begin{array}{l}0 \text { to } 0.032 \mathrm{~kg} / \mathrm{s} \\
(0 \text { to } 250 \mathrm{bm} / \mathrm{h})\end{array}$ & $\begin{array}{l} \pm 0.0013 \mathrm{~kg} / \mathrm{s} \\
( \pm 10 \mathrm{bm} / \mathrm{h})\end{array}$ \\
\hline PT-5 & Steam inlet pressure & 208 & $\begin{array}{l}0 \text { to } 0.69 \mathrm{MPa} \\
(0 \text { to } 100 \mathrm{psia})\end{array}$ & $\begin{array}{l} \pm 0.034 \mathrm{MPa} \\
( \pm 5 \mathrm{psia})\end{array}$ \\
\hline PT -6 & Steam outlet pressure & 209 & $\begin{array}{l}0.069 \text { to } 0.345 \mathrm{MPa} \\
(0 \text { to } 50 \mathrm{psia})\end{array}$ & $\begin{array}{l} \pm 0.017 \mathrm{MPa} \\
( \pm 2.5 \mathrm{psia})\end{array}$ \\
\hline PT -4 & $\begin{array}{l}\text { Steam pressure control, } \\
\text { outlet region }\end{array}$ & 217 & $\begin{array}{l}0 \text { to } 0.69 \mathrm{MPa} \\
(0 \text { to } 100 \mathrm{psia})\end{array}$ & $\begin{array}{l} \pm 0.034 \mathrm{MPa} \\
( \pm 5 \mathrm{psia})\end{array}$ \\
\hline FI-4 & $\begin{array}{l}\text { Reflood coolant, low flow } \\
\text { rate }\end{array}$ & 201 & $\begin{array}{l}0 \text { to } 0.254 \mathrm{~m} / \mathrm{s} \\
(0 \text { to } 10 \mathrm{in.} / \mathrm{s})\end{array}$ & $\pm 5 \%$ \\
\hline $\mathrm{FI}-3$ & $\begin{array}{l}\text { Reflood coolant, high } \\
\text { flow rate }\end{array}$ & 202 & $\begin{array}{l}0.013 \text { to } 0.305 \mathrm{~m} / \mathrm{s} \\
(0.5 \text { to } 12 \mathrm{in.} / \mathrm{s})\end{array}$ & $\pm 5 \%$ \\
\hline FI4b & $\begin{array}{l}\text { Reflood coolant, low } \\
\text { flow rate (backup) }\end{array}$ & 224 & $\begin{array}{l}0 \text { to } 0.254 \mathrm{~m} / \mathrm{s} \\
(0 \text { to } 10 \mathrm{in.} / \mathrm{s})\end{array}$ & $\pm 5 \%$ \\
\hline FI3b & $\begin{array}{l}\text { Reflood coolant, high } \\
\text { flow rate (backup) }\end{array}$ & 223 & $\begin{array}{l}0.013 \text { to } 0.305 \mathrm{~m} / \mathrm{s} \\
(0.5 \text { to } 12 \mathrm{in.} / \mathrm{s})\end{array}$ & $\pm 5 \%$ \\
\hline FE-9 & $\begin{array}{l}\text { Standby reflood coolant } \\
\text { flow }\end{array}$ & 203 & $\begin{array}{l}0.013 \text { to } 0.305 \mathrm{~m} / \mathrm{s} \\
(0.5 \text { to } 12 \mathrm{in.} / \mathrm{s})\end{array}$ & $\pm 10 \%$ \\
\hline TE-17 & $\begin{array}{l}\text { Reflood coolant tempera- } \\
\text { ture control valve inlet }\end{array}$ & 213 & $\begin{array}{l}305 \text { to } 322 \mathrm{~K} \\
\left(90 \text { to } 120^{\circ} \mathrm{F}\right)\end{array}$ & $\pm 5 \%$ \\
\hline$T E-18$ & $\begin{array}{l}\text { Reflood coolant tempera- } \\
\text { ture control valve } \\
\text { out let }\end{array}$ & 214 & $\begin{array}{l}305 \text { to } 322 \mathrm{~K} \\
\left(90 \text { to } 120^{\circ} \mathrm{F}\right)\end{array}$ & $\pm 5 \%$ \\
\hline
\end{tabular}


NRU Reactor Preparations

1. Confirm that two linear rate and two log rate neutron flux detectors (ion chambers) are set and being recorded in the NRU reactor control room.

2. Confirm that the REDACE data will be taken on demand or at a 30-s frequency when requested.

3. Establish mean power, $\log$ rate (at $10 \% / \mathrm{s}$ ), and auxiliary log rate (at $10 \% / s)$ trip set points as required.

4. Confirm that all trip set points are activated, and report to the experiment director when ready for calibration test operation.

DACS Computer Preparations

1. Load the DACS with a Tabeled, certified tape and disks.

2. Start a dummy test, and set the DACS mode to idle.

3. Set the steady-state scan rate at $1 \mathrm{~s}$.

4. Set the transient scan rate at $40 \mathrm{~ms}$.

5. Set the immediate display scan rate at $4 \mathrm{~s}$.

6. Set the graphic display scan rate at $5 \mathrm{~s}$.

7. Format the steady-state immediate display with the sensors iisted in Table 13.

8. Format the transient graphic display.

9. Identify, record (log), and remove the failed sensors from displays, pseudo sensors, and trip circuits.

10. Reset Keithley amplifiers to the 0.1 scale and change SPND coefficients; reduce by a factor of 10 .

11. Report to the experiment director when ready for calibration test. 


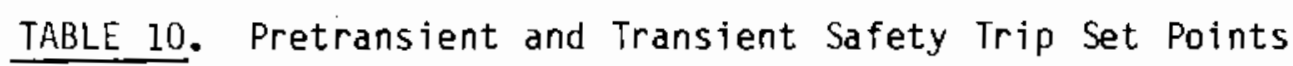

\begin{tabular}{|c|c|c|c|c|}
\hline Parameter & Location & Use & $\begin{array}{c}\text { Operating } \\
\text { Limits } \\
\end{array}$ & $\begin{array}{l}\text { Trip } \\
\text { Set Point }\end{array}$ \\
\hline Hanger tube temperature - high & $\operatorname{LCS}$ & $\begin{array}{l}\text { Pretransient } \\
\text { and transient }\end{array}$ & $691 \mathrm{~K}\left(785^{\circ} \mathrm{F}\right)$ & $839 \mathrm{~K}\left(1050^{\circ} \mathrm{F}\right)$ \\
\hline Outlet pipe temperature - high & LCS & $\begin{array}{l}\text { Pretransient } \\
\text { and transient }\end{array}$ & $672 \mathrm{~K}\left(750^{\circ} \mathrm{F}\right)$ & $700 \mathrm{~K}\left(800^{\circ} \mathrm{F}\right)$ \\
\hline $\begin{array}{l}\text { Fuel cladding temperature - high }(a, b) \\
\text { Level (13) } 76.9,327 \text {, PS-76.9-GR-IR-1 } \\
\text { Level (15) } 97.9,312 \text {, PS-97.9-GR-IR-1 } \\
\text { Level (17) } 118.9,321, \text { PS-118.9-GR-IR-1 }\end{array}$ & $\begin{array}{l}\text { DACS } \\
\text { DACS } \\
\text { DACS }\end{array}$ & Transient & $\begin{array}{ll}1305 \mathrm{~K} & \left(1890^{\circ} \mathrm{F}\right) \\
1305 \mathrm{~K} & \left(1890^{\circ} \mathrm{F}\right) \\
1278 \mathrm{~K} & \left(1840^{\circ} \mathrm{F}\right)\end{array}$ & $\begin{array}{l}1361 \mathrm{~K} \\
1361 \mathrm{~K}\left(1990^{\circ} \mathrm{F}\right) \\
1333 \mathrm{~K}\left(1940^{\circ} \mathrm{F}\right) \\
\left.340^{\circ} \mathrm{F}\right)\end{array}$ \\
\hline Low steam flow trip & LCS & Pretransient & $\begin{array}{l}0.378 \mathrm{~kg} / \mathrm{s} \\
(3000 \mathrm{lbm} / \mathrm{h})\end{array}$ & $\begin{array}{l}0.277 \mathrm{~kg} / \mathrm{s} \\
(2500 \mathrm{lbm} / \mathrm{h})\end{array}$ \\
\hline Accumulator inventory - low & LCS & Transient & $22.7 \mathrm{~kg}(50 \mathrm{lbm})$ & $11.3 \mathrm{~kg}(25 \mathrm{lbm})$ \\
\hline
\end{tabular}

(a) Standard trip set point criteria; see Table 11 for nonfunctional TC criteria.

(b) In use during transient operation only. 
TABLE 11. Standard and Defaulted Safety Trip Set Point Criteria for Operating and Nonfunctional Fuel Cladding Thermocouples

\begin{tabular}{|c|c|c|c|c|}
\hline \multirow[b]{3}{*}{ Qperating Conditions } & \multirow[b]{3}{*}{$\begin{array}{c}\text { Criteria and } \\
\text { Safety Margins } \\
\text { (SM) }\end{array}$} & $\begin{array}{c}\text { Sensors at } \\
\text { Level }(17) 118.9\end{array}$ & $\begin{array}{c}\text { Sensors at } \\
\text { Level (15) } 97.9 \\
\end{array}$ & $\begin{array}{c}\text { Sensor at } \\
\text { Level (13) } 76.9\end{array}$ \\
\hline & & \multicolumn{3}{|c|}{ DACS Themrocouple Nunbers } \\
\hline & & $\begin{array}{l}\text { 321, PS-118.9-GR-IR-1 (a) } \\
\frac{(81,126,92,138)}{\text { Safety Trip }}\end{array}$ & $\begin{array}{l}\text { 312, PS-97.9-GR-IR-1 } \\
\text { (103,25,45,24,2) } \\
\text { set Point Temperatures }\end{array}$ & $\begin{array}{l}\text { 327, PS-76.9-CR-IR-1 } \\
(101,52,67,50) \\
\mathrm{K}(\mathrm{O} F)\end{array}$ \\
\hline $\begin{array}{l}2 \leq \text { Number of operating } \\
\text { TCS on each of } \\
\text { Levels } 76.9,97.9, \\
\text { and } 118.9\end{array}$ & $\begin{array}{c}\text { Standard } \\
S M=56 \mathrm{~K}\left(100^{\circ} \mathrm{F}\right)\end{array}$ & $1333(1940)$ & $1361(1990)$ & $1361(1990)$ \\
\hline $\begin{array}{l}2 \leq \text { Number of operating } \\
\text { TCS on each of } \\
\text { Levels } 97.9 \text { and } \\
118.9\end{array}$ & $\begin{array}{c}\text { Standard } \\
\text { SM }=56 \mathrm{~K}\left(100^{\circ} \mathrm{F}\right)\end{array}$ & $1333(1940)$ & $1361(1990)$ & \\
\hline $\begin{array}{c}2 \leq \text { Number of operating } \\
\text { TCS on each of } \\
\text { Levels } 97.9 \text { and } \\
118.9 \text { or Levels } \\
76.9 \text { and } 118.9\end{array}$ & $\begin{array}{c}\text { Al ternate } \\
S M=84 K\left(150^{\circ} \mathrm{F}\right)\end{array}$ & $1305(1890)$ & $1333(1940)$ & $1333(1940)$ \\
\hline $\begin{array}{l}2 \leq \text { Nmber of operating } \\
\text { TCs on only Level } \\
97.9,118.9, \text { or } \\
76.9\end{array}$ & $\begin{array}{c}\text { Fal lback } \\
S M=111 \mathrm{~K}\left(200^{\circ} \mathrm{F}\right)\end{array}$ & $1277(1840)$ & $1305(1890)$ & $1305 \mathrm{~K}(1890)$ \\
\hline
\end{tabular}

(a) These pseudo sensor data (PSD) are the calculated time-average of the following DACS sensor-numbered data: $81,126,92,138$. 
TABLE 12. Cladding High-Temperature Trip Sensors

\begin{tabular}{|c|c|c|c|c|c|}
\hline \multicolumn{2}{|c|}{ Level } & Sensor & DACS & DACS & DACS \\
\hline (01d) & New & Thermocouples & Sensor Number & Pseudo Sensor & Sensor Number \\
\hline (13) & 76.9 & $\begin{array}{l}T C-76.9-6 C-I R-3 \\
T C-76.9-4 A-I R-3 \\
T C-76.9-5 B-I R-1 \\
T C-76.9-3 F-I R-1\end{array}$ & $\begin{array}{r}101 \\
52 \\
67 \\
50\end{array}$ & PS-76.9-GR-IR-1 & 327 \\
\hline (15) & 97.9 & $\begin{array}{l}T C-97.9-1 C-I R-2 \\
T C-97.9-5 E-I R-2 \\
T C-97.9-5 B-I R-3 \\
T C-97.9-2 B-I R-4 \\
T C-97.9-2 E-I R-1\end{array}$ & $\begin{array}{r}103 \\
53 \\
45 \\
24 \\
2\end{array}$ & PS-97.9-GR -IR-1 & 312 \\
\hline (17) & 118.9 & $\begin{array}{l}\text { TC-118.9-1B-IR-2 } \\
\text { TC-118.9-3B-IR-3 } \\
\text { TC-118.9-4C-IR-8 } \\
\text { TC-118.9-3D-IR-8 }\end{array}$ & $\begin{array}{r}81 \\
126 \\
92 \\
138\end{array}$ & PS-118.9-GR-IR-1 & 32 \\
\hline
\end{tabular}

TABLE 13. DACS Immediate Display Test Sensors

\begin{tabular}{|c|c|c|c|}
\hline Sensor Name & $\begin{array}{c}\text { DACS } \\
\text { Sensor Number }\end{array}$ & Sensor Name & $\begin{array}{c}\text { DACS } \\
\text { Sensor Number }\end{array}$ \\
\hline$T C-76.9-2 C-I R-3$ & 127 & $T C-118.9-3 B-I R-3$ & 126 \\
\hline TC-97.9-2B-IR-C & 9 & $T C-118.9-1 B-I R-2$ & 81 \\
\hline TC-97.9-2C-IR-8 & 55 & TC-118.9-6E-IR-4 & 68 \\
\hline TC-97.9-2D-IR-8 & 119 & $T C-118.9-6 F-S-C$ & 111 \\
\hline$T C-97.9-3 E-I R-8$ & 122 & $T C-97.9-6 \mathrm{~F}-\mathrm{S}-\mathrm{C}$ & 76 \\
\hline$T C-118.9-4 C-I R-8$ & 92 & $T C-139.9-6 F-S-C$ & 129 \\
\hline TC-118.9-3D-IR-8 & 138 & & \\
\hline
\end{tabular}

\section{Reflood Calibration Operating Procedure}

1. Calibrate the reflood prefill controls to fill the test nozzle annulus to Level 0 .

2. Place the DACS in the steady-state mode.

3. Increase test section steam flow to $0.378 \mathrm{~kg} / \mathrm{s}(3000 \mathrm{lbm} / \mathrm{h})$, and control test section backpressure at $0.276 \mathrm{MPa}$ (40 psia). 
4. For successive calibrations, enter the following reflood rates: $0.00508 \mathrm{~m} / \mathrm{s}(0.2 \mathrm{in.} / \mathrm{s}) ; 0.0254 \mathrm{~m} / \mathrm{s}(1.0 \mathrm{in.} / \mathrm{s}) ; 0.0508 \mathrm{~m} / \mathrm{s}(2.0$ $\mathrm{in.} / \mathrm{s})$; and $0.0965 \mathrm{~m} / \mathrm{s}(3.8 \mathrm{in.} / \mathrm{s})$.

5. Print a sensor status report to insure that the test assembly and all TCs are $\geq 422 \mathrm{~K}\left(300^{\circ} \mathrm{F}\right)$.

6. Reproduce the DACS immediate and graphic displays.

7. Turn on the video tape recorder, and zero the counter when loading a new tape.

8. Prefill three times and drain twice.

9. Switch the DACS to the transient mode $20 \mathrm{~s}$ before issuing the verba 1 command "BEGIN THE TRANSIENT" (di rected to the LCS operator), and record the time.

10. LCS operator initiates the calibration transient.

11. Reproduce the DACS immediate and graphic displays as required.

12. Stop the test when reflood water passes the TCs at Leve! (20) 168.7 .

13. Drain the MT-4 test train in L-24, and repeat Steps 2 through 12 until all reflood calibrations are completed.

14. Change the DACS mode to steady state for $5 \mathrm{~min}$ and then to idle.

15. Make a historical request on the DACS graphic display and reproduce copies of the following data:

- reflood rate (DACS sensors 201 and 202)

- steam flow rate (DACS sensor 211 )

- TCs at each level (DACS sensors 289, 291, 296, 299, 302, 304, $327,307,312,310,321,319$, and 320$)$.

16. Print a11 DACS data throughout the transient at 5-s intervals.

17. Turn off the video recorder, and record the counter reading. 
18. Make a tape copy on the DACS and a disk image copy on tape as time permits.

19. Pressurize the 12 test rods to $4.62 \mathrm{MPa}$ (670 psia) using helium; monitor the pressure; and remove the charging system and seal the valves.

20. As necessary, repeat DACS Computer Preparations and Loop System Preparations before proceeding to Pretransient Operating Procedure.

Steam and Water Flow Power Calibration Procedure

1. PIace DACS in the steady-state mode.

2. Increase test section steam flow to $0.378 \mathrm{~kg} / \mathrm{s}(30001 \mathrm{bm} / \mathrm{h})$, and control test section backpressure at $0.276 \mathrm{MPa}$ (40 psia).

3. Manually valve (top of room 501) condensate water from the U-1 boiler feed-water pumps into the reflood accumulator fill line.

4. Bring the NRU reactor to a low neutron power level.

5. LCS operator will set timers and controls for a zero refiood delay and a reflood flow rate of $0.1219 \mathrm{~m} / \mathrm{s}(4.8 \mathrm{in.} / \mathrm{s})$. (a) Values must be approved by the test director.

6. Increase NRU reactor power to nominally 5\% of full power, scan the DACS immediate display for the hottest TCs, and reproduce the display.

7. Insure that the test assembly inlet steam temperature stabilizes at $435 \pm 8 K\left(325 \pm 15^{\circ} \mathrm{F}\right)$.

8. Adjust NRU power to obtain a steady-state temperature increase of 178 $\pm 4 K\left(320 \pm 7^{\circ} \mathrm{F}\right)$ across the test assembly.

9. When steady-state operating conditions have been reached, switch the DACS to the transient mode; $20 \mathrm{~s}$ later, issue the verbal command "BEGIN THE POWER CALIBRATION TRANSIENT" (directed to the LCS operator).

(a) The reflood water flow rate for calorimetry calibration corresponds to a mass $f l o w$ rate of $0.441 \mathrm{~kg} / \mathrm{s}(35001 \mathrm{bm} / \mathrm{h})$. 
10. LCS operator begins the power calibration transient to establish water cooling for power calibration measurements; record time in the Test Parameter Log.

11. Insure that the test assembly inlet water temperature stabilizes at $311 \pm 6 \mathrm{~K}\left(100 \pm 10^{\circ} \mathrm{F}\right)$.

12. When all fuel cladding TCS have quenched, change the DACS operating mode to steady state. Note the control rod movements necessary to maintain a constant NRU reactor power.

13. When steady-state operating conditions have been reached and measurements are completed, shut down the reactor and shut off reflood water flow.

14. Return valve configuration (top of room 501) to normal position supply for the reflood accumulator fill line.

15. Change the DACS operating mode to idle.

16. Make historical requests on the DACS graphic display and reproduce the basic thermal-hydraulic power calibration data.

17. Print all DACS data throughout the transient at 5-s intervals.

Adiabatic Test Procedure

1. Set the protective reactor trip set points for DACS and LCS control (values must be approved and recorded by the test director); see Table 14.

2. LCS operator will set timers for reflood delay times; record values (values must be approved by the test director); see Table 14 .

3. Check accumulator levels (weights), record values in the Test Parameter Log, and drain the test assembly in preparation for steam cooling.

4. Start a new test on the DACS; change DACS mode to steady-state. 
TABLE 14. Adiabatic Experiment Conditions Log

A. Protective trip temperatures for fuel Levels (13) 76.9, (15) 97.9 , and (17) $118.9825 \mathrm{~K}\left(1025^{\circ} \mathrm{F}\right)$

B. Protective trip temperatures for shroud Levels (15) 97.9, (17) 118.9 , and (18) 139.9 825K $\left(1025^{\circ} \mathrm{F}\right)$

C. MT Run

Number

1

\begin{tabular}{|c|c|c|}
\hline \multirow[b]{2}{*}{ Delay Time, s } & \multicolumn{2}{|c|}{ Reflood Rate } \\
\hline & $\mathrm{m} / \mathrm{s}(i n . / \mathrm{s})$ & Duration, $s$ \\
\hline
\end{tabular}

2

$$
\begin{aligned}
& 0.122(4.8) \quad \begin{array}{l}
\text { Continue until steady } \\
\text { state is reached }
\end{array}
\end{aligned}
$$

D. Maximum allowed time (_s) for reflood water to quench sensor _ at Leve 1 not applicable

E. DACS control No

F. Number and settings for DACS control reflood rates not applicable

G. Control function coefficients not applicable

H. Pressurization level $0.101 \mathrm{MPa}(14.7 \mathrm{psia})$, time Room Temperature.

I. Fuel rods pressurized:

Yes $\quad$ No Qty

Sign of $f$

Test Director 
5. Insure that the REDACE scan frequency for NRU data is $30 \mathrm{~s}$.

6. Set the test section steam to $0.378 \mathrm{~kg} / \mathrm{s}(3000 \mathrm{lbm} / \mathrm{h})$ and test section backpressure to $0.28 \mathrm{MPa}$ (40 psia).

7. Increase NRU reactor power to the low neutron level.

8. Before proceeding, NRU reactor operator must acknowledge that the neutron power will not exceed the neutron power level specified by the test director (nominally 5.0\%).

9. Increase NRU neutron power to the level specified. With the power at nominally $5.0 \%$ of the NRU reactor fuel power, scan the DACS immediate display for the hottest IC and reproduce the display.

10. Insure that the test assembly inlet temperature stabilizes at $435 \pm 8 \mathrm{~K}$ $\left(325 \pm 15^{\circ} \mathrm{F}\right)$.

11. Adjust the reactor power to obtain a steady-state $178 \mathrm{~K}\left(320^{\circ} \mathrm{F}\right)$ temperature increase across the test assembly.

12. Reproduce the DACS immediate and graphic displays.

13. Activate the video tape recorder.

14. Prefill a total of three times and drain twice. 0isplay test assembly temperatures on the DACS graphic display.

15. Change to the transient operating mode on the DACS; $20 \mathrm{~s}$ later, issue the verbal command "BEGIN THE TRANSIENT" (directed to the LCS operator); and record the time in the Test Parameter Log.

16. The LCS operator begins the transient.

17. When the fuel cladding temperature reaches $825 \mathrm{~K}\left(1025^{\circ} \mathrm{F}\right)$ or the protective trip set points on the DACS and LCS, the NRU reactor is shut down or automatically tripped and full reflood flow quenches the test assembly.

18. Repeat Steps 1 through 17 until the steam flow and NRU reactor power provide the required $8.3 \mathrm{~K} / \mathrm{s}\left(15^{\circ} \mathrm{F} / \mathrm{s}\right)$ full-temperature ramp rate. Leave the test assembly filled with coolant water. 
TABLE 15. LOCA Experiment Conditions Log

A. Protective trip temperatures for fuel Levels (13) 76.9, (15) 97.9 , and (17) 118.9

B. Protective trip temperatures for shroud Levels (15) 97.9, (17) 118.9 , and (18) $139.9850 \mathrm{~K}\left(1070^{\circ} \mathrm{F}\right)$ for Level 118.9

C. MT Run

$\begin{array}{ccc}\text { Delay Time, } s & \frac{\text { Reflood Rate }}{\text { m/s(in./s) }} & \text { Duration, s } \\ & & \\ & 0.203(8) & 6 \\ 0.102(4) & 6 \\ & 0.025(1) & 3\end{array}$

D. Maximum allowed time s) for reflood water to quench sensor at Level not applicable

E. DACS control Yes, $72 \mathrm{~s}$ after start of transient

F. Number and settings for DACS control reflood rates

\begin{tabular}{|c|c|c|c|}
\hline Setting & $\begin{array}{l}\text { Reflood Rate, } \\
\mathrm{m} / \mathrm{s}(\text { in. } / \mathrm{s})\end{array}$ & Setting & $\begin{array}{l}\text { Reflood Rate, } \\
\mathrm{m} / \mathrm{s}(\mathrm{in} . / \mathrm{s})\end{array}$ \\
\hline 1 & 0 & 6 & $0.0152(0.60)$ \\
\hline 2 & $0.0051(0.20)$ & 7 & $0.0203(0.80)$ \\
\hline 3 & $0.0076(0.30)$ & 8 & $0.0254(1.00)$ \\
\hline 4 & $0.0089(0.35)$ & 9 & $0.0508(2.00)$ \\
\hline 5 & $0.0102(0.40)$ & & \\
\hline
\end{tabular}

G. Control function coefficients $\Delta T=1.0, T^{\prime}=10.0, T^{\prime \prime}=6.0$ $\Delta T=$ absolute value of minimum allowable temperature deviation $T^{\prime}=$ differential temperature gain coefficient $T^{\prime \prime}$ = second derivative of temperature gain coefficient

H. Pressurization leve $14.62 \mathrm{MPa}(670$ psia), time 10:30 p.m., and temperature $296 \mathrm{~K}\left(73^{\circ} \mathrm{F}\right)$

I. Fuel rods pressurized:

$\mathrm{X}$ Yes ${ }^{\mathrm{No}}+12$ Qty

Sign off

Test Director 


\section{Pretransient Operating Procedure}

1. Set the protective reactor trip set points for DACS and LCS control (values must be approved and recorded by the test director); see Table 15.

2. LCS operator will set timers for reflood delay times (Table 8); record values (values must be approved by the test director); see Table 15.

3. Check accumulator levels (weights), record values, and drain the test assembly in preparation for steam cooling.

4. Start a new test on the DACS; change DACS mode to steady-state.

5. Insure that the REDACE scan frequency for NRU data is $30 \mathrm{~s}$.

6. Set the test section steam to $0.378 \mathrm{~kg} / \mathrm{s}(30001 \mathrm{bm} / \mathrm{h})$ and test section backpressure to $0.28 \mathrm{MPa}$ (40 psia).

7. Increase NRU reactor power to the low neutron level.

8. Before proceeding, NRU reactor operator must acknowledge that neutron power will not exceed percent power level specified by test director (nominally $5.0 \%$ ).

9. Increase NRU reaccor power to the level specified. With the reactor power at nominally $5.0 \%$ of NRU full power, scan the DACS immediate display for the hottest TC and reproduce the display.

10. Insure that the test assembly inlet temperature stabilizes at $435 \pm 8 \mathrm{~K}$ $\left(325 \pm 15^{\circ} \mathrm{F}\right)$.

11. If necessary, adjust the reactor power to obtain a steady-state $178 \mathrm{~K}$ $\left(320^{\circ} \mathrm{F}\right)$ temperature increase across the test assembly; begin collecting traveling fission chamber data.

12. Check the peak cladding temperature, steam flow, test assembly inlet temperature, and outlet pressure. Complete fission chamber data records and attach them to the Test Parameter Log.

13. Reproduce the DACS immediate and graphic displays.

14. Activate the video tape recorder. 
Transient Operating Procedure

1. Prefill the inlet annulus a total of three times and drain it twice. Display test assembly temperatures on the DACS graphic display.

2. Change to the transient operating mode on the DACS; $20 \mathrm{~s}$ later, issue the verbal command "BEGIN THE TRANSIENT" (directed to the LCS operator); and record the time in the Test Parameter Log.

3. The LCS operator begins the transient.

4. When fuel cladding temperatures exceed $1200 \mathrm{~K}\left(1700^{\circ} \mathrm{F}\right)$, the LCS will revert reflood flow control to the DACS-selected rates--0 to $0.00508 \mathrm{~m} / \mathrm{s}(0.2 \mathrm{in.} / \mathrm{s})$.

5. Complete thermal-hydraulic measurements at stabilized peak cladding temperatures of $1089 \mathrm{~K}\left(1500^{\circ} \mathrm{F}\right)$ and $1200 \mathrm{~K}\left(1700^{\circ} \mathrm{F}\right)(\sim 2$ to $3 \mathrm{~min}$ each).

6. Shut down the NRU reactor when measurements are complete, and use full reflood flow to quench the test assembly.

7. Shut off reflood water flow only after upper TCs have quenched.

8. Record reflood water used (accumulator weight difference) in the Test Parameter Log.

9. Insure that tripping the reactor has returned control to the DACS (transient forcing signal $\# 257=0$ ).

10. Return the DACS mode to steady-state for $5 \mathrm{~min}$ and then to idle, ending the data record.

11. End the test on the DACS. Verify that the Test Parameter Log is completed.

12. Turn off the video tape recorder, and record the location.

13. Copy the following historical data on the DACS:

- Make a hard copy of the hottest pseudo sensors at Levels (15) 97.9 and (17) 118.9.

- Plot data for the following sensors: 289, 29u, and 211. 



\section{REFERENCES}

1. Hann, C. R., et al. July 1979. Program Plan for LOCA Simulation in the National Research Universal (NRU) Reactor. PNL-3056, Pacific Northwest Laboratory, Richland, Washington.

2. Bennett, W. D., et al. February 1981. Safety Analys is Report: Loss-ofCoolant Accident Simulations in the National Research Universal Reactor. NUREG/CR-1208, PNL-3093, Pacific Northwest Laboratory, Richland, Washington.

3. Russcher, G. E., et al. June 1983. Experiment Operations Plan for the MT-3 Experiment in the NRU Reactor. NUREG/CR-2599, PNL-4190, Pacific Northwest Laboratory, Richland, Washington.

4. Russcher, G. E., et al. April 1981. Experiment Operations Plan for a Loss-of-Coolant Accident Simulation in the National Research Universal Reactor. NUREG/CR-1735, PNL-3551, Pacific Northwest Laboratory, Richland, Washington.

5. Mohr, C. L., et al. April 1981. Prototypic Thermal-Hydraulic Experiment in NRU to Simulate Loss-of-Coolant Accidents. NUREG/CR-1882, PNL-3681, Pacific Northwest Laboratory, Richland, Washington.

6. Atfield, M. D., memorandum to NRU Superintendent. October 2, 1980. "Reactor Loading for Battelle LOCA Simulations Tests." Chalk River Nuclear Laboratories, Chalk River, Ontario, Canada.

7. Axford, 0. J., I. C. Martin, and S. J. McAuTey. 1980. Final Safety and Hazards Analysis for the Battelle LOCA Simulation Tests in the NRU Reactor. AECL 7153, Chalk River Nuclear Laboratories, Chalk River, Ontario, Canada. 

APPENDIX A

TEST PARAMETER LOG 


\section{APPENDIX A}

TEST PARAMETER LOG

LOCA Simulation in NRU

Run number:

Date:

\subsection{PRECONDITIONING ACTIVITIES}

Date:

Time:

Data stored on data tape number:

Disk number:

1.1 Power calculations (attach to $\log$ )

1.2 Traveling fission chamber data (attach to $\mathrm{log}$ )

\subsection{PRETRANSIENT ACTIVITIES}

Tape number:

Disk configuration: DPO

DP1

DP1F

\subsection{Operation summary}

\section{PARAMETER/UNITS}

Steam flow rate, $1 \mathrm{bm} / \mathrm{h}$

Steam inlet temperature, ${ }^{\circ} \mathrm{F}$

Maximum fuel cladding temperatures, ${ }^{\circ} \mathrm{F}$

Sensor name

Sensor name

Sensor name

Total test assembly $\Delta \mathrm{T},{ }^{\circ} \mathrm{F}$

outlet pressure, psia
SPECIFIED VALUE

(ACCEPTED RANGE) ACTUAL VALUE

$325( \pm 15)$

800 (NA)
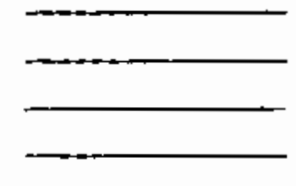

$320( \pm 10)$

$40( \pm 5 \%)$ 
2.2 Print all sensor data with DACS in steady-state mode; review.

2.3 Protective trip set points (pretransient and transient)

\begin{tabular}{l} 
PARAMETER/UNITS \\
\hline Hanger tube temperature - high, ${ }^{\circ} \mathrm{F}$ \\
Outlet pipe temperature - high, ${ }^{\circ} \mathrm{F}$ \\
Steam flow - low, lbm/h \\
Fuel cladding temperature, ${ }^{\circ} \mathrm{F}$ \\
Level (17) $118.9-$ high \\
Level (15) $97.9-$ high \\
Level (13) $76.9-$ high \\
Time to quench Level (20) 168.7
\end{tabular}

2.4 Traveling fission chamber data (attach to $\log$ )

3.0 SPECIAL COMMENTS ON RUN CONDITIONS:

3.1 Preparations:

3.2 Pretransient:

3.3 Transient: 
3.4 Post-transient:

4.0 CONDITIONS CAUSING RUN TERMINATION:

5.0 SPECIAL CONDITIONS TO BE CONSIDERED IN THE ANALYSIS OF THE TEST RUN: 
6.0 CONDITIONS THAT MAY CAUSE THE TEST TO BE INVALID:

7.0 INSTRUMENTATION FAILURES BEFORE TEST: 


\subsection{INSTRUMENTATION FAILURES AFTER TEST TERMINATION:}

9.0 GENERAL COMMENTS ON TEST:

$$
\text { A. } 5
$$



APPENDIX B

EXPERIMENT CONOITIONS LOG 


\section{APPENDIX B}

EXPERIMENT CONDITIONS LOG

A. Protective trip temperatures for fuel Levels (13) 76.9, (15) 97.9 , and (17) $11 \mathrm{~B} .9$

B. Protective trip temperatures for shroud Levels (15) 97.9, (17) 118.9 , and (18) 139.9

C. MT Run Number Delay Time, s mls (in./s) Duration, s

D. Maximum allowed time s) for reflood water to quench sensor at Level

E. DACS control

F. Number and settings for DACS control reflood rates

G. Control function coefficients

H. Pressurization Level , time and temperature

I. Fuel rods pressurized:

Yes ${ }^{\text {No _._. }}{ }^{\text {Qty }}$

Sign $0 f f$

Test Director 



\section{DISTRIBUTION}

No. of

Copies

OFFSITE

U.S. Nuclear Regulatory Commission

Division of Technical Information and Document Control

7920 Norfolk Avenue

Bethesda, MO 20014

$20 \mathrm{R}$. Van Houten

U.S. Nuclear Regulatory Commission

Fuel Systems Research Branch Washington, DC 20555

FORE IGN

6 D. T. Nishimura

Chalk River Nuclear Laboratories

Chalk River, Ontario

Canada
No. of

Copies

ONSITE

50 Pacific Northwest Laboratory

W. J. Bailey

L. W. Cannon

S. K. Edler

M. D. Freshley

R. L. Goodman

C. R. Hann

G. M. Hesson

U. P. Jenquin

L. L. King

R. R. Lewis (2)

R. K. Marshall

P. N. McDuffie

L.J. Parchen (5)

W. N. Rausch

G. E. Russcher (16)

B. J. Webb

N. J. Wildung

C. L. Wilson (5)

M. C. Wismer

Publishing Coordination (2)

Technical Information (5) 



\begin{tabular}{|c|c|}
\hline $\begin{array}{l}\text { U.S. NUCLEAR REGULATORY COMHISSION } \\
\text { BIBLIOGRAPHIC DATA SHEET }\end{array}$ & $\begin{array}{l}\text { 1. AEPORT NUMEE R (A ssigned by DOC) } \\
\text { NUREG/CR-288T } \\
\text { PNL-4293 }\end{array}$ \\
\hline 4. TITLE AND SUBTITLE (Addo Volume No, if eporoorise) & 2. (Leave blenk) \\
\hline $\begin{array}{l}\text { EXPERIMENT OPERATIONS PLAN FOR THE MT-4 EXPERIMENT IN } \\
\text { THE NRU REACTOR }\end{array}$ & 3. RECIPIENT'S ACCESSION NO. \\
\hline $\begin{array}{l}\text { 7. AUTHOR(S) G.E. Russcher, C.L. Wilson, L.J. Parchen, } \\
\text { R.K. Marshall, G. M. Hesson, B.J. Webb, M.D. Freshley }\end{array}$ & \begin{tabular}{|c|c|} 
5. DATE AEPORT COMPLETED \\
MONTH \\
MaY & TYEAR \\
\end{tabular} \\
\hline \multirow{3}{*}{$\begin{array}{l}\text { Pacific Northwest Laboratory } \\
\text { P.0. Box } 999 \\
\text { Richland, WA } 99352\end{array}$} & \begin{tabular}{l|l} 
DATE REPOAT ISSUED & \\
MONTH & \\
JUNE & YEAF \\
\end{tabular} \\
\hline & 6. (Leave bisnk) \\
\hline & 8. (Leave biank) \\
\hline \multirow{2}{*}{$\begin{array}{l}\text { 12. SPONSORING ORGANIZATION NAME AND MAILING ADORESS \#nciude Zio Code) } \\
\text { Division of ACCident Evaluation } \\
\text { Office of Nuclear Regulatory Research } \\
\text { U.S. Nuclear Regulatory Commission } \\
\text { Washington, D.C. } 20555\end{array}$} & 10. PROJECT/TASK/WOAK UNIT NO. \\
\hline & $\begin{array}{r}\text { 11. FIN NO. } \\
\text { B2277 }\end{array}$ \\
\hline
\end{tabular}

15. SUPPLEMENTARY NOTES

14. (Lewe ofomk)

16. ABSTAACT (200 words or less)

A series of thema? -hydraulic and cladding materials deformation experiments were conducted using light-water reactor fuel bundles as part of the Pacific Northwest Laboratory Loss-of-Coolant Accident (LOCA) Simulation Program. This report is the formal operations plan for MT-4--the fourth materials deformation experiment conducted in the National Research Universal (NRU) reactor, Chalk River, Ontario, Canada. A major objective of MT-4 was to simulate a pressurized water reactor LOCA that could induce fuel rod ciadding deformation and rupture due to a short-term adiabatic transient and a peak fuel cladding temperature of $1200 \mathrm{~K}\left(1700^{\circ} \mathrm{F}\right)$.

17. KEY WOADS ANO DOCUMENT ANALYSIS

thermal-hydraulic loop control system data acquisition and control system light-water reactor fuel bundles cladding deformation and rupture 17a. DESCRIPTORS

experiment operations plan

loss-of-coolant accident simulation National Research Universal (NRU) reactor

170. IOENTIFIERSIOPEN-ENOEO TERMS 
